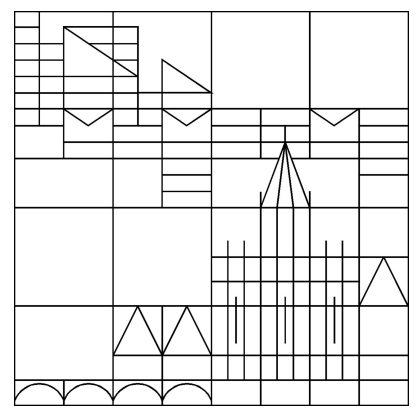

\title{
Decay rates of semilinear viscoelastic systems in weighted spaces
}

\author{
Reinhard Racke \\ Belkacem Said-Houari
}

Konstanzer Schriften in Mathematik

Nr. 275, Dezember 2010

ISSN 1430-3558

Konstanzer Online-Publikations-System (KOPS)

URN: http://nbn-resolving.de/urn:nbn:de:bsz:352-opus-126623

URL: http://kops.ub.uni-konstanz.de/volltexte/2010/12662/ 



\title{
Decay rates of semilinear viscoelastic systems in weighted spaces
}

\author{
REINHARD RACKE* AND BELKACEM SAID-HOUARI $^{\dagger}$ \\ Department of Mathematics and Statistics \\ University of Konstanz \\ 78457 Konstanz, Germany
}

\begin{abstract}
In this paper we consider a class of second-order hyperbolic system which describe viscoelastic materials and we extend the results in the recent papers [4] and [1]. More precisely, if we take the initial data $\left(u_{0}, u_{1}\right) \in\left(H^{s+1}\left(\mathbb{R}^{N}\right) \cap L^{1, \gamma}\left(\mathbb{R}^{N}\right)\right) \times\left(H^{s}\left(\mathbb{R}^{N}\right) \cap L^{1, \gamma}\left(\mathbb{R}^{N}\right)\right)$ with $\gamma \in[0,1]$, then we can derive faster decay estimates than those given in [1] and in [4] for both dissipative structure or regularityloss type models. To this end, we will first transform our problem into Fourier space, then, by using the pointwise estimate derived in [4] combined with a device to treat the Fourier transform in the low frequency region, we succeed in proving the optimal decay results to the solutions of our problem. Finally, we use these decay estimates of the linear problem combined with the weighted energy method introduced by Todorova and Yordanov [27] to tackle a semilinear problem.
\end{abstract}

Keywords: Hyperbolic system, viscoelasticity, energy method, memory kernel, polynomial decay, decay rate.

\section{Introduction}

In this paper, we are concerned with the following second order hyperbolic systems with dissipation:

$$
u_{t t}-\sum_{j, k=1}^{N} B^{j k} u_{x_{j} x_{k}}+\sum_{j, k=1}^{N} K^{j k} * u_{x_{j} x_{k}}+L u_{t}=f(u), \quad x=\left(x_{1}, \ldots, x_{N}\right) \in \mathbb{R}^{N}, t \geq 0
$$

where $*$ denotes the convolution with respect to $t$, that is

$$
(g * u)(t)=\int_{0}^{t} g(t-\tau) u(\tau) d \tau,
$$

where $g$ will be exponentially decaying, and $f(u)=-|u|^{p-1} u(p \geq 1)$.

We consider the following initial data

$$
u(x, 0)=u_{0}(x), \quad u_{t}(x, 0)=u_{1}(x), \quad x \in \mathbb{R}^{N},
$$

${ }^{*}$ E-mail: reinhard.racke@uni-konstanz.de,

†E-mail: belkacem.said-houari@uni-konstanz.de 
where $u$ is an $m$-vector function of $x, B^{j k}$ are $m \times m$ real constant matrices satisfying $\left(B^{j k}\right)^{T}=B^{k j}$ for each $j$ and $k$ and $K^{j k}(t)$ are $m \times m$ matrix functions of $t \geq 0$ satisfying $\left(K^{j k}(t)\right)^{T}=K^{k j}(t)$ for each $j, k$ and $t \geq 0$, and $L$ is an $m \times m$ constant matrix.

For $f=0$, i.e. the linear problem (1.1)-(1.2) has been considered recently in [4] and, by using the energy method in the Fourier space, the authors have obtained a decay rate of the form

$$
\left\|\partial_{x}^{k} u_{t}(t)\right\|_{2}+\left\|\partial_{x}^{k+1} u(t)\right\|_{2} \leq C(1+t)^{-N / 4-k / 2}\left\|u_{1}\right\|+C(1+t)^{-N / 4-k / 2-1 / 2}\left\|u_{0}\right\|_{1},
$$

provided that the initial data $\left(u_{0}, u_{1}\right) \in H^{s+1}\left(\mathbb{R}^{N}\right) \cap L^{1}\left(\mathbb{R}^{N}\right) \times H^{s}\left(\mathbb{R}^{N}\right) \cap L^{1}\left(\mathbb{R}^{N}\right)$ where $0 \leq k \leq s$. They also showed that, by introducing the operator $(1-\Delta)^{-\theta / 2}, \theta>0$ in front one of the dissipative terms, the decay structure of the system is of the regularity-loss type, see (5.1) in [4].

For $m=1, B^{j k}=\delta_{j k}$, and $K^{j k}(t)=\delta_{j k} g(t)$ problem (1.1) takes the form

$$
u_{t t}-\Delta u+\int_{0}^{t} g(t-s) \Delta u(s) d s+u_{t}+|u|^{p-1} u=0
$$

where $\delta_{j k}$ is the Kronecker symbol.

Problems similar to (1.3) arise in viscoelasticity and in systems governing the longitudinal motion of a viscoelastic configuration obeying a nonlinear Boltzmann's model where the constitutive relation between the stress $\sigma(x, t)$ and the strain $\varepsilon(x, t)$ is of the form

$$
\sigma(x, t)=c \varepsilon(x, t)-\int_{0}^{\infty} m(s) \varepsilon(x, t-s) d s
$$

where $c$ is a positive constant and $m$ is positive, decreasing, integrable function satisfies

$$
c-\int_{0}^{\infty} m(s) d s>0 .
$$

In the unbounded domain case, there is very little in the literature that discusses the global existence and decay properties of solution even for the linear problem.

Hrusa and Nohel [10] considered a problem similar to (1.3) in $\mathbb{R}$ as a model for the motion of an unbounded, homogeneous, viscoelastic bar. More precisely, they investigated the problem

$$
u_{t t}=\phi\left(u_{x}(x, t)\right)_{x}+\int_{0}^{t} a^{\prime}(t-s) \psi\left(u_{x}(x, s)\right)_{x} d s+f(x, t)
$$

which can be obtained by considering a nonlinear generalization of the constitutive equation (1.4) of the form

$$
\sigma(x, t)=\phi(\varepsilon(x, t))-\int_{0}^{\infty} m(s) \psi(\varepsilon(x, t-s)) d s
$$

where $\phi$ and $\psi$ are smooth functions. Under reasonable conditions on $\phi$ and $\psi$ and assuming the smallness condition on the initial data, they proved the existence of a unique global classical solution of the Cauchy problem (1.5). They also established an asymptotic decay result but no rate of decay was given. Dassios and Zafiropoulus [3] investigated the model of the linear viscoelastic system in $\mathbb{R}^{3}$ and proved, by using the Fourier transform in the space variable, that the decay is of order $t^{-3 / 2}$ provided that the relaxation is an exponential function like $\mu_{0} e^{-\mu_{1} t},\left(\mu_{0}, \mu_{1}>0\right)$. The result in [3] has been extended by Muñoz Rivera [23] 
to $\mathbb{R}^{N}$ by showing that the decay rate is of order $t^{-N / 2}$, provided the relaxation is any function which decays exponentially to zero. Recently, Kafini and Messaoudi [15] have considered the Cauchy problem

$$
u_{t t}-\Delta u+\int_{0}^{t} g(t-s) \Delta u(s) d s=0
$$

and showed a polynomial decay of the energy associated to problem (1.6), provided the kernel $g$ decays exponentially and the initial data are compactly supported. But no precise decay rate was given in [15].

Conti et al [1] looked at the linear problem (1.6) in the so-called history space framework, and showed that if the kernel $g$ decays exponentially, then the solution of (1.6) satisfies

$$
\left\|u_{t}(t)\right\|_{2}+\|\nabla u(t)\|_{2} \leq C(1+t)^{-N / 4}
$$

While if $g$ decays polynomially, i.e.

$$
g(s) \leq C(1+s)^{-1-p}, \quad p>0
$$

then the solution of $u$ of (1.6) satisfies

$$
\left\|u_{t}(t)\right\|_{2}^{2}+\|\nabla u(t)\|_{2}^{2} \leq \begin{cases}C(1+t)^{-\min \{p, N p /(2 p+2)\}} & \text { if } 2 p \neq N-2 \\ C(1+t)^{-p} \log (2+t) & \text { if } 2 p=N-2 .\end{cases}
$$

Both estimates (1.7) and (1.8) hold for initial data satisfying $u_{0} \in H^{1}\left(\mathbb{R}^{N}\right) \cap L^{1}\left(\mathbb{R}^{N}\right)$ and $u_{1} \in L^{2}\left(\mathbb{R}^{N}\right) \cap$ $L^{1}\left(\mathbb{R}^{N}\right)$.

Observe that if $g$ vanishes identically, then problem (1.2), (1.3) reduces to the damped wave equation:

$$
\begin{cases}u_{t t}-\Delta u+u_{t}+|u|^{p-1} u=0, & t>0, x \in \mathbb{R}^{N}, \\ u(0, x)=u_{0}(x), u_{t}(0, x)=u_{1}(x), & x \in \mathbb{R}^{N} .\end{cases}
$$

Problem (1.9) has been extensively studied and several results concerning existence and asymptotic behavior have been established, see $[6,7,8,13,17,19,20]$ and references therein. By using the energy method combined with $L^{p}-L^{q}$ estimates, Kawashima et al [17] showed that if

$$
1+\frac{4}{N}<p<\frac{N+2}{N+3}, \text { for } N=3 \text { or } 1+\frac{4}{N}<p<\infty, \text { for } N=1,2
$$

then the solution $u(t, x)$ of (1.9) decays as

$$
\|u\|_{2} \leq C t^{-\frac{N}{2}\left(\frac{1}{r}-\frac{1}{2}\right)}
$$

provided that the initial data

$$
\left(u_{0}, u_{1}\right) \in\left(H^{1}\left(\mathbb{R}^{N}\right) \cap L^{r}\left(\mathbb{R}^{N}\right)\right) \times\left(L^{2}\left(\mathbb{R}^{N}\right) \cap L^{r}\left(\mathbb{R}^{N}\right)\right)
$$

for $1 \leq r \leq 2$. Based on the result of [17], Karch [16] showed that the solution of (1.9) behaves as that of the corresponding diffusive equation

$$
u_{t}-\Delta u+|u|^{p-1} u=0 .
$$

More precisely, he proved that

$$
\left\|u(t)-\theta_{0} G(t, .)\right\|_{2}=O\left(t^{-N / 4}\right)
$$


where

$$
\theta_{0}=\int_{\mathbb{R}^{N}}\left(u_{0}+u_{1}\right)(x) d x-\int_{0}^{\infty} \int_{\mathbb{R}^{N}}|u|^{p-1} u(t, x) d x d t
$$

and

$$
G(t, x)=(4 \pi)^{-N / 2} e^{\frac{-|x|^{2}}{4 t}} .
$$

Recently, Nishihara and Zhao [20] have investigated the problem (1.9) and showed that for $1<p<1+2 / N$ the solution of (1.9) decays as

$$
\left(\|u(t)\|_{p},\|\nabla u(t)\|_{2}\right)=O\left(t^{-\frac{1}{\rho-1}+\frac{N}{2 \rho}}, t^{-\frac{1}{\rho-1}+\frac{N}{4}-\frac{1}{2}}\right), \quad 1 \leq p \leq \rho+1 .
$$

The decay result in [20] has been obtained without any smallness condition on the initial data. Instead, and in order to apply the weighted $L^{2}$-energy method, the initial data in [20] are assumed to satisfy $e^{\beta|x|^{2}}\left(u_{0}, \nabla u_{0}, u_{1}\right)$ $\in L^{2}\left(\mathbb{R}^{N}\right)$ for some $\beta>0$. When the absorbing term $|u|^{p-1} u$ in (1.9) is replaced by a forcing term of the form $-|u|^{p}$ the situation is more delicate. More precisely, Todorova and Yordanov [27] considered the Cauchy problem

$$
u_{t t}-\Delta u+u_{t}=|u|^{p}
$$

and showed that the value $p_{0}=1+2 / N$ is the critical exponent of (1.12). In other words, for $p>1+2 / N$, they proved that any solution of (1.12) with sufficiently small initial data exists globally in time and decays as

$$
\left\|\left(u_{t}, \nabla u\right)\right\|_{2}=O\left(t^{-\frac{N}{4}-\frac{1}{2}}\right) .
$$

On the other hand if $1<p \leq 1+2 / N$, then every solution of (1.12) with initial data having positive average value blows up in finite time. In their result it is essentially used that the initial data associated to (1.12) have a compact support, which leads to the finite propagation speed property of the wave. The result in [27] has been improved by Ikehata and Tanizawa [14], in which the authors used a weight function, which modifies that of Todorova and Yordanov [27] and helped them to remove the compactness assumptions on the support of the initial data. The decay rates (1.13) given in [27] and [14] have been improved in [12] to be of the form

$$
\left(\|u\|_{2},\left\|u_{t}\right\|_{2},\|\nabla u\|_{2}\right)=O\left(t^{-\frac{N}{4}-\frac{\gamma}{2}}, t^{-\frac{N}{4}-\frac{\gamma}{2}-\frac{1}{2}}, t^{-\frac{N}{4}-\frac{\gamma}{2}-\frac{1}{2}}\right)
$$

provided that the initial data $\left(u_{0}, u_{1}\right) \in\left(H^{1}\left(\mathbb{R}^{N}\right) \cap L^{1, \gamma}\left(\mathbb{R}^{N}\right)\right) \times\left(L^{2}\left(\mathbb{R}^{N}\right) \cap L^{1, \gamma}\left(\mathbb{R}^{N}\right)\right)$ with $\gamma \in[0,1]$ and $\int_{\mathbb{R}^{N}} u_{i}(x) d x=0, i=0,1$.

The plan of this paper is as follows: In section 2, we fix notations and for the convenience of the reader, we recall, without proofs, some useful lemmas. In section 3, we treat problem (1.1)-(1.2) and we show that by restricting ourselves to initial data $\left(u_{0}, u_{1}\right) \in\left(H^{s+1}\left(\mathbb{R}^{N}\right) \cap L^{1, \gamma}\left(\mathbb{R}^{N}\right)\right) \times\left(H^{s}\left(\mathbb{R}^{N}\right) \cap L^{1, \gamma}\left(\mathbb{R}^{N}\right)\right)$ with $\gamma \in[0,1]$, then we can derive faster decay estimates than those given in [4]. In fact, by transforming our problem into Fourier space, using the pointwise estimates derived in [4] and adapting the devise introduced by Ikehata in [12], to treat the Fourier transform in the low frequency region, we succeed to improve the decay rate given in [4] by $t^{-\gamma / 2}, \gamma \in[0,1]$. In section 4 , and as it was shown in [4], by introducing the operator $(1-\Delta)^{-\theta / 2},(\theta>0)$ to weaken the linear damping term or the viscoelastic damping in problem (1.1), then the decay structure of the corresponding systems is of regularity-loss type [9, 11]. Once again, our decay estimates in this case are better than those given in [4]. In section 5, we investigate problem (1.3) and show that even in the absence of the frictional damping $u_{t}$, the damping given by the viscoelastic term still strong enough to stabilize the solution of (1.3) with the same decay rate as in the presence of $u_{t}$. (See Remark 7.1 and Remark 7.2 for more comments). Our result in this section extends the result of [1] in 
which the decay rates for both exponential and polynomial kernels have been improved by assuming further restrictions on the initial data. Section 6 is devoted to analyze the asymptotic behavior of the semilinear problem (1.3). More precisely, we use the decay estimates obtained in section 3 for the linear problem combined with the weighted energy method introduced by Todorova and Yordanov [27] to obtain some optimal decay estimates for (1.3), this is the contents of subsection 6.1. In subsection 6.2, and by using the energy method, we obtain some decay estimates for (1.3) in the subcritical region $1<p<1+4 / N$. This result extends the one given in [20] to the viscoelastic wave equation. Finally, in section 7, we give some comments and compare the obtained results. We note here that the compactness assumption on the support of the initial data is unnecessary in this paper, and our results hold without it.

\section{Preliminaries}

As in [4], and in order to make the appropriate assumptions, we define the following real symmetric matrices:

$$
B_{\omega}=\sum_{j, k=1}^{N} B^{j k} \omega_{j} \omega_{k}, \quad K_{\omega}(t)=\sum_{j, k=1}^{N} K^{j k}(t) \omega_{j} \omega_{k}
$$

for $\omega=\left(\omega_{1}, \ldots, \omega_{N}\right) \in S^{N-1}$ and $t \geq 0$. In order to state an prove our main result, and following [4], we make the following assumptions

(A1) $B_{\omega}$ is real symmetric and positive definite for each $\omega \in S^{N-1}, K_{\omega}(t)$ is real symmetric and nonnegative definite for each $\omega \in S^{N-1}$ and for all $t \geq 0$, and $L$ is real symmetric and nonnegative definite.

(A2) $B_{\omega}-\mathscr{K}_{\omega}(t)$ is real symmetric and positive definite for each $\omega \in S^{N-1}$ uniformly in $t \geq 0$, where $\mathscr{K}_{\omega}(t)=\int_{0}^{t} K_{\omega}(s) d s$.

(A3) $K_{\omega}(0)+L$ is real symmetric and positive definite for each $\omega \in S^{N-1}$.

(A4) There are positive constants $C_{0}$ and $c_{0}$ such that

$$
-C_{0} K_{\omega}(t) \leq K_{\omega}^{\prime}(t) \leq-c_{0} K_{\omega}(t),
$$

and

$$
-C_{0} K_{\omega}(t) \leq K_{\omega}^{\prime \prime}(t) \leq C_{0} K_{\omega}(t)
$$

for each $\omega \in S^{N-1}$ and $t \geq 0$, where $K_{\omega}^{\prime}(t)=\partial_{t} K_{\omega}(t)$ and $K_{\omega}^{\prime \prime}(t)=\partial_{t}^{2} K_{\omega}(t)$.

Now, we introduce some notations to be used throughout this paper.

Throughout this paper, $\|\cdot\|_{q}$ and $\|\cdot\|_{H^{l}}$ stand for the $L^{q}\left(\mathbb{R}^{N}\right)$-norm $(2 \leq q \leq \infty)$ and the $H^{l}\left(\mathbb{R}^{N}\right)$-norm. Also, for $\gamma \in[0,+\infty)$, we define the weighted function space $L^{p, \gamma}\left(\mathbb{R}^{N}\right), 1 \leq p<\infty, N \geq 1$, as follows: $u \in L^{p, \gamma}\left(\mathbb{R}^{N}\right)$ iff

$$
\|u\|_{p, \gamma}=\int_{\mathbb{R}^{N}}(1+|x|)^{\gamma}|u(x)|^{p} d x<+\infty .
$$

Concerning the function $g$ introduced in (1.3), we assume the following:

(G1) $g: \mathbb{R}_{+} \rightarrow \mathbb{R}_{+}$is a $C^{1}$ function satisfying

$$
g(0)>0, \quad 1-\int_{0}^{\infty} g(s) d s=l>0 .
$$


(G2) There exists $\zeta>0$ such that

$$
g^{\prime}(t) \leq-\zeta g(t), \quad \forall t \geq 0
$$

Let us also denote $\hat{f}=\mathscr{F}(f)$ be the Fourier transform of $f$ :

$$
\hat{f}(\xi)=\mathscr{F}(f)(\xi)=\int_{\mathbb{R}^{N}} f(x) e^{-i \xi x} d x,
$$

and let $\mathscr{F}^{-1}(f)$ be the inverse Fourier transform of $f$.

Let us introduce the following notations:

$$
\begin{aligned}
& (\varphi * \psi)(t):=\int_{0}^{t} \varphi(t-\tau) \psi(\tau) d \tau \\
& (\varphi \diamond \psi)(t):=\int_{0}^{t} \varphi(t-\tau)|\psi(t)-\psi(\tau)|^{2} d \tau, \\
& (\varphi \circ \psi)(t):=\int_{0}^{t} \varphi(t-\tau) \int_{\mathbb{R}^{N}}|\psi(t)-\psi(\tau)|^{2} d x d \tau .
\end{aligned}
$$

The following lemma was introduced in [24, Lemma 3.2] and we will use it later in this paper.

Lemma 2.1 For any function $\varphi \in C^{1}(\mathbb{R})$ and any $\psi \in H^{1}(0, T)$, we have

$$
\begin{aligned}
(\varphi * \psi)(t) \psi_{t}(t)= & -\frac{1}{2} \varphi(t)|\psi(t)|^{2}+\frac{1}{2}\left(\varphi^{\prime} \diamond \psi\right)(t) \\
& -\frac{1}{2} \frac{d}{d t}\left\{(\varphi \diamond \psi)(t)-\left(\int_{0}^{t} \varphi(\tau) d \tau\right)|\psi(t)|^{2}\right\} .
\end{aligned}
$$

The following lemma was introduced and proved in [1, Lemma 1.2] and [25, Lemma 3.5].

Lemma 2.2 Let $k_{0} \geq 1, m_{0}>0$ and $t>0$, then we have

$$
\int_{0}^{1} \frac{r^{k_{0}-1}}{(1+r t)^{m_{0}}} d r \leq \begin{cases}C t^{-\min \left\{m_{0}, k_{0}\right\}}, & \text { if } m_{0} \neq k_{0} \\ C t^{-k_{0}} \log t, & \text { if } m_{0}=k_{0}\end{cases}
$$

where $C$ is a positive constant.

\section{Decay estimates for $f=0$}

In this section, we consider problem (1.1)-(1.2) for $f=0$ (i.e. the linear problem) and we prove some optimal decay estimates. In fact, if we restrict the initial data $u_{0} \in\left(H^{s+1}\left(\mathbb{R}^{N}\right) \cap L^{1, \gamma}\left(\mathbb{R}^{N}\right)\right)$ and $u_{1} \in$ $\left(H^{s}\left(\mathbb{R}^{N}\right) \cap L^{1, \gamma}\left(\mathbb{R}^{N}\right)\right), \gamma \in[0,1]$, then we can derive faster decay estimates than those in [4]. More precisely, our first result reads as follows.

Theorem 3.1 Let $\gamma \in[0,1]$. Let $s$ be a nonnegative integer and assume that $u_{0} \in H^{s+1}\left(\mathbb{R}^{N}\right) \cap L^{1, \gamma}\left(\mathbb{R}^{N}\right)$ and $u_{1} \in H^{s}\left(\mathbb{R}^{N}\right) \cap L^{1, \gamma}\left(\mathbb{R}^{N}\right)$. Suppose that the conditions (A1)-(A4) hold. Then the corresponding solution of 
problem (1.1)-(1.2) $(f=0)$ satisfying the decay estimates

$$
\begin{aligned}
& \left\|\partial_{x}^{k} u_{t}(t)\right\|_{2}+\left\|\partial_{x}^{k+1} u(t)\right\|_{2} \\
& \leq C(1+t)^{-N / 4-\frac{k+\gamma}{2}}\left\|u_{1}\right\|_{1, \gamma}+C(1+t)^{-N / 4-k / 2}\left(\left|\int_{\mathbb{R}^{N}} u_{1}(x) d x\right|\right) \\
& +C(1+t)^{-N / 4-\frac{k+\gamma}{2}-1 / 2}\left\|u_{0}\right\|_{1, \gamma}+C(1+t)^{-N / 4-k / 2-1 / 2}\left(\left|\int_{\mathbb{R}^{N}} u_{0}(x) d x\right|\right) \\
& +C e^{-c t}\left(\left\|\partial_{x}^{k} u_{1}\right\|_{2}+\left\|\partial_{x}^{k+1} u_{0}\right\|_{2}\right)
\end{aligned}
$$

for $k$ with $0 \leq k \leq s$, where $C$ and $c$ are two positive constants.

Remark 3.2 The estimate (3.1) shows that by taking the initial data $u_{0} \in H^{s+1}\left(\mathbb{R}^{N}\right) \cap L^{1, \gamma}\left(\mathbb{R}^{N}\right)$ and $u_{1} \in$ $H^{s}\left(\mathbb{R}^{N}\right) \cap L^{1, \gamma}\left(\mathbb{R}^{N}\right)$, such that $\int_{\mathbb{R}^{N}} u_{i}(x) d x=0, i=0,1$, then the decay rates given in [4, Theorem 3.1] can be improved by $t^{-\gamma / 2}, \gamma \in[0,1]$.

Applying the Fourier transform to our problem (1.1)-(1.2) yields

$$
\left\{\begin{array}{l}
\hat{u}_{t t}+|\xi|^{2} B_{\omega} \hat{u}-|\xi|^{2}\left(K_{\omega} * \hat{u}\right)+L \hat{u}_{t}=0 \\
\hat{u}(\xi, 0)=\hat{u}_{0}(\xi), \quad \hat{u}_{t}(\xi, 0)=\hat{u}_{1}(\xi)
\end{array}\right.
$$

where $B_{\omega}$ and $K_{\omega}$ are given in (2.1) with $\omega=\xi /|\xi|$.

In order to proof Theorem 3.1, we recall the following result from [4, Proposition 3.2]. The proof of the following Proposition is carried out by using the energy method in the Fourier space.

Proposition 3.3 Assume the same conditions as in Theorem 3.1. Then the solution of problem (1.1)-(1.2) satisfies the pointwise estimate

$$
\left|\hat{u}_{t}(\xi, t)\right|^{2}+|\xi|^{2}|\hat{u}(\xi, t)|^{2} \leq C e^{-c \rho(\xi) t}\left(\left|\hat{u}_{1}(\xi)\right|^{2}+|\xi|^{2}\left|\hat{u}_{0}(\xi)\right|^{2}\right)
$$

where $\rho(\xi)=\xi^{2} /\left(1+|\xi|^{2}\right)$ and $C$ and c are two positive constants.

\section{Proof of Theorem 3.1.}

The prove of Theorem 3.1 is reduced through the Fourier transform to the analysis of the behavior of the spectral parameter $\xi$ near the origin $\xi=0$. That is to say, in order to get better decay estimates, we have to improve the decay estimate of the low frequency part, since the high frequency part has an exponential decay rate. Indeed, from the Plancherel theorem, we can write

$$
\begin{aligned}
\left\|\partial_{x}^{k} u_{t}(t)\right\|_{2}^{2}+\left\|\partial_{x}^{k+1} u(t)\right\|_{2}^{2} & =\int_{\mathbb{R}^{N}}|\xi|^{2 k}\left(\left|\hat{u}_{t}(\xi, t)\right|^{2}+|\xi|^{2}|\hat{u}(\xi, t)|^{2}\right) d \xi \\
& \leq C \int_{\mathbb{R}^{N}}|\xi|^{2 k} e^{-c \rho(\xi) t}\left(\left|\hat{u}_{1}(\xi)\right|^{2}+|\xi|^{2}\left|\hat{u}_{0}(\xi)\right|^{2}\right) d \xi
\end{aligned}
$$

where we have used (3.2).

Next, we divide the integral in the right-hand side of (3.3) into two parts: the low frequency part $(|\xi| \leq 1)$ and the high frequency part $(|\xi| \geq 1)$. So, we have

$$
\begin{aligned}
I & =\int_{\mathbb{R}^{N}}|\xi|^{2 k} e^{-c \rho(\xi) t}\left(\left|\hat{u}_{1}(\xi)\right|^{2}+|\xi|^{2}\left|\hat{u}_{0}(\xi)\right|^{2}\right) d \xi \\
& =\int_{|\xi| \leq 1}+\int_{|\xi| \geq 1}=I_{1}+I_{2} .
\end{aligned}
$$

For the low frequency part $I_{1}$, we have the following estimate: 
Lemma 3.4 Let us suppose that $\gamma \in[0,1]$. Then the following estimate holds

$$
\begin{aligned}
& I_{1} \leq C(1+t)^{-N / 2-(k+\gamma)}\left\|u_{1}\right\|_{1, \gamma}^{2}+C(1+t)^{-N / 2-k}\left(\left|\int_{\mathbb{R}^{N}} u_{1}(x) d x\right|\right)^{2} \\
& +C(1+t)^{-N / 2-(k+\gamma)-1}\left\|u_{0}\right\|_{1, \gamma}^{2}+C(1+t)^{-N / 2-k-1}\left(\left|\int_{\mathbb{R}^{N}} u_{0}(x) d x\right|\right)^{2} .
\end{aligned}
$$

Proof. From (3.4) we have

$$
\begin{aligned}
I_{1} & =\int_{|\xi| \leq 1}|\xi|^{2 k} e^{-c \rho(\xi) t}\left(\left|\hat{u}_{1}(\xi)\right|^{2}+|\xi|^{2}\left|\hat{u}_{0}(\xi)\right|^{2}\right) d \xi \\
& =\int_{|\xi| \leq 1}|\xi|^{2 k} e^{-c \rho(\xi) t}\left|\hat{u}_{1}(\xi)\right|^{2} d \xi+\int_{|\xi| \leq 1}|\xi|^{2 k+2} e^{-c \rho(\xi) t}\left|\hat{u}_{0}(\xi)\right|^{2} d \xi \\
& =J_{1}+J_{2} .
\end{aligned}
$$

Our goal now is to estimate $J_{1}$. To this end, we begin by analyzing the term $\left|\hat{u}_{1}(\xi)\right|$ in $J_{1}$. Indeed, we have (see [12, Lemma 3.1])

$$
\begin{aligned}
\left|\hat{u}_{1}(\xi)\right| & =\left|\int_{\mathbb{R}^{N}} e^{-i x \cdot \xi} u_{1}(x) d x\right| \\
& \leq \int_{\mathbb{R}^{N}}|\cos (x . \xi)-1|\left|u_{1}(x)\right| d x+\int_{\mathbb{R}^{N}}|\sin (x \cdot \xi)|\left|u_{1}(x)\right| d x+\left|\int_{\mathbb{R}^{N}} u_{1}(x) d x\right| .
\end{aligned}
$$

Since

$$
\left\{\begin{array}{l}
K_{\gamma}=\sup _{\theta \neq 0} \frac{|1-\cos \theta|}{|\theta|^{\gamma}}<+\infty \\
M_{\gamma}=\sup _{\theta \neq 0} \frac{\sin \theta}{|\theta|^{\gamma}}<+\infty
\end{array}\right.
$$

for $0 \leq \gamma \leq 1$. Then we deduce

$$
\left|\hat{u}_{1}(\xi)\right| \leq C_{\gamma}|\xi|^{\gamma}\left\|u_{1}\right\|_{1, \gamma}+\left|\int_{\mathbb{R}^{N}} u_{1}(x) d x\right|
$$

with $C_{\gamma}=K_{\gamma}+M_{\gamma}$.

Consequently, using (3.7), we obtain

$$
J_{1} \leq C\left\|u_{1}\right\|_{1, \gamma}^{2} \int_{|\xi| \leq 1}|\xi|^{2(k+\gamma)} e^{-c \rho(\xi) t} d \xi+\left|\int_{\mathbb{R}^{N}} u_{1}(x) d x\right|^{2} \int_{|\xi| \leq 1}|\xi|^{2 k} e^{-c \rho(\xi) t} d \xi
$$

Since, in the low frequency part $(|\xi| \leq 1)$, we have $\rho(\xi)=\xi^{2} /\left(1+|\xi|^{2}\right) \geq|\xi|^{2} / 2$, then (3.8) becomes

$$
\begin{aligned}
J_{1} & \leq C\left\|u_{1}\right\|_{1, \gamma}^{2} \int_{|\xi| \leq 1}|\xi|^{2(k+\gamma)} e^{-c_{1}|\xi|^{2} t} d \xi+\left|\int_{\mathbb{R}^{N}} u_{1}(x) d x\right|^{2} \int_{|\xi| \leq 1}|\xi|^{2 k} e^{-c_{1}|\xi|^{2} t} d \xi \\
& =C\left\|u_{1}\right\|_{1, \gamma}^{2} \int_{0}^{1}|\xi|^{2(k+\gamma)+N-1} e^{-c_{1}|\xi|^{2} t} d|\xi|+\left|\int_{\mathbb{R}^{N}} u_{1}(x) d x\right|^{2} \int_{0}^{1}|\xi|^{2 k+N-1} e^{-c_{1}|\xi|^{2} t} d|\xi|
\end{aligned}
$$


where $c_{1}=c / 2$. By exploiting the following inequality

$$
\int_{0}^{1}|\xi|^{\sigma} e^{-c t|\xi|^{2}} d|\xi| \leq C(1+t)^{-(\sigma+1) / 2}
$$

we deduce

$$
J_{1} \leq C(1+t)^{-(k+\gamma)-N / 2}\left\|u_{1}\right\|_{1, \gamma}^{2}+(1+t)^{-k-N / 2}\left|\int_{\mathbb{R}^{N}} u_{1}(x) d x\right|^{2} .
$$

With the same kind of arguments, we analyze $J_{2}$ and deduce

$$
J_{2} \leq C(1+t)^{-N / 2-(k+\gamma)-1}\left\|u_{0}\right\|_{1, \gamma}^{2}+C(1+t)^{-N / 2-k-1}\left(\left|\int_{\mathbb{R}^{N}} u_{0}(x) d x\right|\right)^{2} .
$$

Consequently, the result of Lemma 3.4 holds from (3.6), (3.10) and (3.11).

Now, to complete the proof of Theorem 3.1, it is suffices to estimate $I_{2}$. Indeed for the integral $I_{2}$, we have the same estimate as in [4], that is

$$
I_{2} \leq C e^{-c t}\left(\left\|\partial_{x}^{k} u_{1}\right\|_{2}+\left\|\partial_{x}^{k+1} u_{0}\right\|_{2}\right) .
$$

Thus, the result of Theorem 3.1 is verified.

\section{Decay estimate for the regularity-loss type}

In this section, and following [4], we introduce the operator $(1-\Delta)^{-\theta / 2},(\theta>0)$ to weaken the damping term $L u_{t}$ or the viscoelastic damping $\sum_{j, k=1}^{N} K^{j k} * u_{x_{j} x_{k}}$ in problem (1.1). Namely, we consider the following two problems

$$
\left\{\begin{array}{l}
u_{t t}-\sum_{j, k=1}^{N} B^{j k} u_{x_{j} x_{k}}+(1-\Delta)^{-\theta / 2} \sum_{j, k=1}^{N} K^{j k} * u_{x_{j} x_{k}}+L u_{t}=0, \quad x=\left(x_{1}, \ldots, x_{N}\right) \in \mathbb{R}^{N}, t \geq 0 \\
u(x, 0)=u_{0}(x), \quad u_{t}(x, 0)=u_{1}(x), \quad x \in \mathbb{R}^{N}
\end{array}\right.
$$

and

$$
\left\{\begin{array}{l}
u_{t t}-\sum_{j, k=1}^{N} B^{j k} u_{x_{j} x_{k}}+\sum_{j, k=1}^{N} K^{j k} * u_{x_{j} x_{k}}+(1-\Delta)^{-\theta / 2} L u_{t}=0, \quad x=\left(x_{1}, \ldots, x_{N}\right) \in \mathbb{R}^{N}, t \geq 0 \\
u(x, 0)=u_{0}(x), \quad u_{t}(x, 0)=u_{1}(x), \quad x \in \mathbb{R}^{N}
\end{array}\right.
$$

Once again, we improve the decay rate obtained in [4, Theorem 5.1]. As, we will prove later, introducing the weak damping in (4.1) or (4.2) affects only the high frequency part. So, we will get only a polynomial decay rate of the high frequency part instead of the exponential rate obtained in Theorem 3.1 for this part.

Our main result in this section reads as follows. 
Theorem 4.1 Let $\gamma \in[0,1]$. Let $s$ be a nonnegative integer and let $\theta>0$. Assume that $u_{0} \in H^{s+1}\left(\mathbb{R}^{N}\right) \cap$ $L^{1, \gamma}\left(\mathbb{R}^{N}\right)$ and $u_{1} \in H^{s}\left(\mathbb{R}^{N}\right) \cap L^{1, \gamma}\left(\mathbb{R}^{N}\right)$. Suppose that the conditions (A1)-(A4) hold. Then the corresponding solution of problem (4.1) or (4.2) satisfies the decay estimate

$$
\begin{aligned}
& \left\|\partial_{x}^{k} u_{t}(t)\right\|_{2}+\left\|\partial_{x}^{k+1} u(t)\right\|_{2} \\
& \leq C(1+t)^{-N / 4-\frac{k+\gamma}{2}}\left\|u_{1}\right\|_{1, \gamma}+C(1+t)^{-N / 4-k / 2}\left(\left|\int_{\mathbb{R}^{N}} u_{1}(x) d x\right|\right) \\
& +C(1+t)^{-N / 4-\frac{k+\gamma}{2}-1 / 2}\left\|u_{0}\right\|_{1, \gamma}+C(1+t)^{-N / 4-k / 2-1 / 2}\left(\left|\int_{\mathbb{R}^{N}} u_{0}(x) d x\right|\right) \\
& +C(1+t)^{-l / \theta}\left(\left\|\partial_{x}^{k+l} u_{1}\right\|_{2}+\left\|\partial_{x}^{k+l+1} u_{0}\right\|_{2}\right)
\end{aligned}
$$

for nonnegative integers $k$ and $l$ with $k+l \leq s$, where $C$ is a positive constant.

Remark 4.2 Once again, the result of Theorem 4.1 improves the one of [4, Theorem 5.1], especially if we restrict ourselves to the case when $u_{0} \in H^{s+1}\left(\mathbb{R}^{N}\right) \cap L^{1, \gamma}\left(\mathbb{R}^{N}\right)$ and $u_{1} \in H^{s}\left(\mathbb{R}^{N}\right) \cap L^{1, \gamma}\left(\mathbb{R}^{N}\right)$ rather than the situation considered in [4], that is $u_{0} \in H^{s+1}\left(\mathbb{R}^{N}\right) \cap L^{1}\left(\mathbb{R}^{N}\right)$ and $u_{1} \in H^{s}\left(\mathbb{R}^{N}\right) \cap L^{1}\left(\mathbb{R}^{N}\right)$, we are able to prove the better decay estimate

$$
\begin{aligned}
\left\|\partial_{x}^{k} u_{t}(t)\right\|_{2}+\left\|\partial_{x}^{k+1} u(t)\right\|_{2} \leq & C(1+t)^{-N / 4-\frac{k+\gamma}{2}}\left\|u_{1}\right\|_{1, \gamma}++C(1+t)^{-N / 4-\frac{k+\gamma}{2}-1 / 2}\left\|u_{0}\right\|_{1, \gamma} \\
& +C(1+t)^{-l / \theta}\left(\left\|\partial_{x}^{k+l} u_{1}\right\|_{2}+\left\|\partial_{x}^{k+l+1} u_{0}\right\|_{2}\right)
\end{aligned}
$$

provided that $\int_{\mathbb{R}^{N}} u_{i}(x) d x=0, i=0,1$.

To prove Theorem 4.1, we proceed as in the previous section, we write first our problems (4.1) and (4.2) in the Fourier space, and we use the pointwise estimates obtained in [4] to get our desired result. As, we have said before, the key ingredient in the proof is an idea used by Ikehata [12] for the simple wave equation and developed by Said-Houari [26] for the p-system with damping.

Applying the Fourier transform to both problems (4.1) and (4.2), we get respectively

$$
\left\{\begin{array}{l}
\hat{u}_{t t}+|\xi|^{2} B_{\omega} \hat{u}-|\xi|^{2}\left(1+|\xi|^{2}\right)^{-\theta / 2}\left(K_{\omega} * \hat{u}\right)+L \hat{u}_{t}=0 \\
\hat{u}(\xi, 0)=u_{0}(\xi), \quad \hat{u}_{t}(\xi, 0)=u_{1}(\xi)
\end{array}\right.
$$

and

$$
\left\{\begin{array}{l}
\hat{u}_{t t}+|\xi|^{2} B_{\omega} \hat{u}-|\xi|^{2}\left(K_{\omega} * \hat{u}\right)+\left(1+|\xi|^{2}\right)^{-\theta / 2} L \hat{u}_{t}=0 \\
\hat{u}(\xi, 0)=u_{0}(\xi), \quad \hat{u}_{t}(\xi, 0)=u_{1}(\xi)
\end{array}\right.
$$

where $B_{\omega} \hat{u}$ and $K_{\omega}$ are defined in (2.1) with $\omega=\xi /|\xi|$.

By using the energy method in the Fourier space, we have (see [4, Proposition 5.2])

Proposition 4.3 Assume the same conditions of Theorem 4.1. Then, the solutions of problem (4.4) or (4.5) satisfies the pointwise estimate

$$
\left|\hat{u}_{t}(\xi, t)\right|^{2}+|\xi|^{2}|\hat{u}(\xi, t)|^{2} \leq C e^{-c \rho_{\theta}(\xi) t}\left(\left|\hat{u}_{1}(\xi)\right|^{2}+|\xi|^{2}\left|\hat{u}_{0}(\xi)\right|^{2}\right),
$$

where $\rho_{\theta}(\xi)=\xi^{2} /\left(1+|\xi|^{2}\right)^{1+\theta / 2}$ and $C$ and $c$ are two positive constants. 
Proof of Theorem 4.1 As in the proof of Theorem 3.1, Plancherel theorem and (4.6) give us the following estimate:

$$
\begin{aligned}
\left\|\partial_{x}^{k} u_{t}(t)\right\|_{2}^{2}+\left\|\partial_{x}^{k+1} u(t)\right\|_{2}^{2} & \leq C \int_{\mathbb{R}^{N}}|\xi|^{2 k} e^{-c \rho_{\theta}(\xi) t}\left(\left|\hat{u}_{1}(\xi)\right|^{2}+|\xi|^{2}\left|\hat{u}_{0}(\xi)\right|^{2}\right) d \xi \\
& =\int_{|\xi| \leq 1}+\int_{|\xi| \geq 1}=\hat{I}_{1}+\hat{I}_{2} .
\end{aligned}
$$

Since for the region $(|\xi| \leq 1)$ we have $\rho_{\theta}(\xi) \geq c|\xi|^{2}$, then

$$
\hat{I}_{1} \leq C \int_{|\xi| \leq 1}|\xi|^{2 k} e^{-c|\xi|^{2}}\left(\left|\hat{u}_{1}(\xi)\right|^{2}+|\xi|^{2}\left|\hat{u}_{0}(\xi)\right|^{2}\right) d \xi,
$$

which can be estimated exactly as $I_{1}$ in Lemma 3.4. Therefore, we have

$$
\begin{aligned}
& \hat{I}_{1} \leq C(1+t)^{-N / 2-(k+\gamma)}\left\|u_{1}\right\|_{1, \gamma}^{2}+C(1+t)^{-N / 2-k}\left(\left|\int_{\mathbb{R}^{N}} u_{1}(x) d x\right|\right)^{2} \\
& +C(1+t)^{-N / 2-(k+\gamma)-1}\left\|u_{0}\right\|_{1, \gamma}^{2}+C(1+t)^{-N / 2-k-1}\left(\left|\int_{\mathbb{R}^{N}} u_{0}(x) d x\right|\right)^{2} .
\end{aligned}
$$

For, the term $\hat{I}_{2}$, we obtain by the same method used in [4] the estimate

$$
\hat{I}_{2} \leq C(1+t)^{-2 l / \theta}\left(\left\|\partial_{x}^{k+l} u_{1}\right\|_{2}^{2}+\left\|\partial_{x}^{k+l+1} u_{0}\right\|_{2}^{2}\right) .
$$

Consequently, the estimate (4.3) follows from (4.7), (4.8) and (4.9). This complete the proof of Theorem 4.1.

\section{The viscoelastic wave equation without linear damping}

In this section, we consider problem (1.3) without the linear damping $u_{t}$. In this case the dissipativity of the system is entirely contained in the convolution term. We show that this dissipation carried out by the memory term is strong enough to produce a decay result of the solution with the same rate as in the case of the presence of $u_{t}$. Our result in this section extends that of [1]. Let us consider the problem

$$
\begin{cases}u_{t t}-\Delta u+\int_{0}^{t} g(t-s) \Delta u(s) d s=0, & t>0, x \in \mathbb{R}^{N}, \\ u(0, x)=u_{0}(x), u_{t}(0, x)=u_{1}(x), & x \in \mathbb{R}^{N} .\end{cases}
$$

Under further restrictions on the initial data, we prove that the decay rate given in [1] can be also improved by $t^{-\gamma / 2}, \gamma \in[0,1]$, when the kernel decays exponentially or polynomially.

\subsection{The kernel $g$ decays exponentially}

In this subsection, we prove a result similar to Theorem 3.1 which extends Theorem 2.1 in [1]. Our result reads as follows. 
Theorem 5.1 Let $\gamma \in[0,1]$. Assume that $u_{0} \in H^{1}\left(\mathbb{R}^{N}\right) \cap L^{1, \gamma}\left(\mathbb{R}^{N}\right)$ and $u_{1} \in L^{2}\left(\mathbb{R}^{N}\right) \cap L^{1, \gamma}\left(\mathbb{R}^{N}\right)$ and suppose that

$$
g(s) \leq \tilde{\lambda} e^{-\tilde{\delta} s}, \quad \forall s \geq 1
$$

for some $\tilde{\lambda}>0$ and $\tilde{\delta}>0$. Then

$$
\begin{aligned}
\left\|u_{t}\right\|_{2}+\|\nabla u\|_{2} \leq & \hat{C}(1+t)^{-N / 4-\frac{\gamma}{2}}\left\|u_{1}\right\|_{1, \gamma}+\hat{C}(1+t)^{-N / 4}\left(\left|\int_{\mathbb{R}^{N}} u_{1}(x) d x\right|\right) \\
& +\hat{C}(1+t)^{-N / 4-\frac{\gamma}{2}-1 / 2}\left\|u_{0}\right\|_{1, \gamma}+\hat{C}(1+t)^{-N / 4-1 / 2}\left(\left|\int_{\mathbb{R}^{N}} u_{0}(x) d x\right|\right) \\
& +\hat{C} e^{-\hat{c} t}\left(\left\|u_{1}\right\|_{2}+\left\|\nabla u_{0}\right\|_{2}\right)
\end{aligned}
$$

where $\hat{C}$ and $\hat{c}$ are two positive constants.

Proof. To prove Theorem 5.1, we follow the ideas in [1], and we apply the method in section 3 and 4 to treat the low frequency part. By making a change of variables, problem (5.1) can be rewritten as

$$
u_{t t}-\Delta u+\int_{0}^{t} g(s) \Delta u(t-s) d s=0
$$

Following the idea of Dafermos [2], setting $u(x, t)=0$ when $t<0$, and introducing the auxiliary variable

$$
\eta^{t}(x, s)=u(x, t)-u(x, t-s), \quad s \in \mathbb{R}^{+}
$$

which implies

$$
\left\{\begin{array}{l}
\eta_{t}^{t}(x, s)+\eta_{s}^{t}(x, s)=u_{t}(x, t) \\
\eta^{t}(x, 0)=0 \\
\eta^{0}(x, s)=u_{0}
\end{array}\right.
$$

Consequently equation (5.3) takes the form

$$
\begin{cases}u_{t t}-l \Delta u+\int_{0}^{\infty} g(s) \Delta \eta^{t}(x, s) d s=0, & t>0, x \in \mathbb{R}^{N}, \\ u(0, x)=u_{0}(x), \quad u_{t}(0, x)=u_{1}(x), & x \in \mathbb{R}^{N} .\end{cases}
$$

Taking the Fourier transform of (5.4)-(5.5), we obtain

$$
\begin{cases}\hat{u}_{t t}(t)-l|\xi|^{2} \hat{u}(t)+|\xi|^{2} \int_{0}^{\infty} g(s) \hat{\eta}^{t}(s) d s=0, & t>0, \\ \hat{\eta}_{t}^{t}(x, s)+\hat{\eta}_{s}^{t}(x, s)=\hat{u}_{t}(x, t), & t>0, \\ \hat{u}(0)=\hat{u}_{0}, \hat{u}_{t}(0)=\hat{u}_{1}, \hat{\eta}^{0}(s)=\hat{u}_{0} . & \end{cases}
$$

The energy function associated to (5.6) is given by (see [1])

$$
\hat{E}(\xi, t)=l|\xi|^{2}|\hat{u}(\xi, t)|^{2}+\left|\hat{u}_{t}(\xi, t)\right|^{2}+|\xi|^{2} \int_{0}^{\infty} g(s)\left|\hat{\eta}^{t}(\xi, s)\right|^{2} d s
$$

with

$$
\hat{E}(\xi, 0)=|\xi|^{2}\left|\hat{u}_{0}(\xi)\right|^{2}+\left|\hat{u}_{1}(\xi)\right|^{2}
$$


Following the same steps as in [1], we get for $|\xi| \geq 1$

$$
\int_{|\xi| \geq 1} \hat{E}(\xi, t) d \xi \leq \hat{k}^{2} e^{-\hat{\varepsilon} t} \int_{|\xi| \geq 1} \hat{E}(\xi, 0) d \xi \leq \hat{k}^{2} e^{-\hat{\varepsilon} t} E(0) e^{-\hat{\varepsilon} t}
$$

for some $\hat{k} \geq 1$ and for all $\hat{\varepsilon}>0$ small enough, where

$$
E(t)=\left\|u_{t}\right\|_{2}^{2}+l\|\nabla u\|_{2}^{2}+\int_{\mathbb{R}^{N}} \int_{0}^{\infty} g(s)\left|\nabla \eta^{t}(x, s)\right|^{2} d s d x .
$$

The Plancherel theorem gives

$$
E(t)=\int_{\mathbb{R}^{N}} \hat{E}(\xi, t) d \xi
$$

As, we have said before, since the high frequency part decays exponentially, then to get better decay estimates, we manage to improve the decay rate of the low frequency part. Indeed, from [1], we have for $|\xi|<1$,

$$
\begin{aligned}
\int_{|\xi|<1} \hat{E}(\xi, t) d \xi & \leq \hat{k}^{2} \int_{|\xi|<1} \hat{E}(\xi, 0) e^{-\hat{\varepsilon}|\xi|^{2} t} d \xi \\
& =\hat{k}^{2} \int_{|\xi|<1} e^{-\hat{\varepsilon}|\xi|^{2} t}\left(|\xi|^{2}\left|\hat{u}_{0}(\xi)\right|^{2}+\left|\hat{u}_{1}(\xi)\right|^{2}\right) d \xi
\end{aligned}
$$

By the same method, as in the proof of Lemma 3.4, we get for $0 \leq \gamma \leq 1$,

$$
\left|\hat{u}_{i}(\xi)\right| \leq C_{\gamma}|\xi|^{\gamma}\left\|u_{i}\right\|_{1, \gamma}+\left|\int_{\mathbb{R}^{N}} u_{i}(x) d x\right|, \quad i=0,1 .
$$

Inserting (5.12) into (5.11), we get

$$
\begin{aligned}
\int_{|\xi|<1} \hat{E}(\xi, t) d \xi \leq & C_{\gamma} \hat{k}^{2} \int_{|\xi|<1} e^{-\hat{\varepsilon}|\xi|^{2} t}\left(|\xi|^{2 \gamma+2}\left\|u_{0}\right\|_{1, \gamma}^{2}+|\xi|^{2 \gamma}\left\|u_{1}\right\|_{1, \gamma}^{2}\right) d \xi \\
& +\hat{k}^{2}\left|\int_{\mathbb{R}^{N}} u_{0}(x) d x\right|^{2} \int_{|\xi|<1} e^{-\hat{\varepsilon}|\xi|^{2} t}|\xi|^{2} d \xi \\
& +\hat{k}^{2}\left|\int_{\mathbb{R}^{N}} u_{1}(x) d x\right| \int_{|\xi|<1} e^{-\hat{\varepsilon}|\xi|^{2} t} d \xi
\end{aligned}
$$

Passing to polar coordinates, using (3.9), (5.8) and (5.10) then (5.2) holds. This completes the proof of Theorem 5.1.

\subsection{The kernel $g$ decays polynomially}

In this subsection, we assume that our kernel $g$ decays polynomially and we extend the result due to Conti et al [1, Thoerem 3.2] by showing that for initial data in some weighted spaces, we get better decay rates. Our result is summarized in the next theorem.

Theorem 5.2 Let $\gamma \in[0,1]$. Assume that $u_{0} \in H^{1}\left(\mathbb{R}^{N}\right) \cap L^{1, \gamma}\left(\mathbb{R}^{N}\right)$ and $u_{1} \in L^{2}\left(\mathbb{R}^{N}\right) \cap L^{1, \gamma}\left(\mathbb{R}^{N}\right)$ satisfying $\int_{\mathbb{R}^{N}} u_{i}(x) d x=0, i=0,1$, and suppose that

$$
g(s) \leq \hat{\zeta}(1+s)^{-1-p}, \quad \forall s \geq 1
$$

for some $\hat{\zeta}>0$ and $p>0$. Then $E(t)$ decays polynomially depending on $p, \gamma$ and $N$ as given in (5.25), (5.26), (5.27), (5.28) and (5.29). 
The proof of Theorem 5.2 is based on the following Lemma. This Lemma itself extends [1, Lemma 7.1]. As in [1], let us first define

$$
\Psi_{p}(\xi, t)=\int_{0}^{t} \Upsilon_{p}(t-s)|\hat{u}(\xi, s)|^{2} d s
$$

where

$$
\Upsilon_{p}=\frac{1}{(1+t)^{p}}, \quad p<\infty
$$

Then, we have:

Lemma 5.3 For any fixed $\xi \in \mathbb{R}^{N}$, we have

$$
\frac{d}{d t}\left[|\xi|^{2} \tilde{g}(\xi) \Psi_{p}(\xi, t)\right]+\frac{c}{[\hat{E}(\xi, 0)]^{q-1}}\left[|\xi|^{2} \tilde{g}(\xi) \Psi_{p}(\xi, t)\right]^{q} \leq \frac{\tilde{g}}{l} \hat{E}(\xi, t),
$$

where

$$
\tilde{g}(\xi)=\frac{|\xi|^{2}}{1+|\xi|^{2}}
$$

and $c$ is generic positive constant.

Proof. Let $\mathscr{L}$ be the function defined in [1, Lemma 5.2], then the following properties hold, (see [1, Lemma 5.3])

$$
\left\{\begin{array}{l}
\frac{d}{d t} \mathscr{L}(\xi, t)+2 \varepsilon_{0} \hat{E}(\xi, t) \leq 0 \\
\frac{1+|\xi|^{2}}{\hat{k}|\xi|^{2}} \hat{E}(\xi, t) \leq \mathscr{L}(\xi, t) \leq \frac{\hat{k}\left(1+|\xi|^{2}\right)}{|\xi|^{2}} \hat{E}(\xi, t)+\hat{k}|\xi|^{2} \Psi_{p}(\xi, t) \\
\mathscr{L}(\xi, 0) \leq \frac{\hat{k}\left(1+|\xi|^{2}\right)}{|\xi|^{2}} \hat{E}(\xi, 0)
\end{array}\right.
$$

for some $\varepsilon_{0}>0$ and $\hat{k}>1$. Differentiating $\Psi_{p}$ with respect to $t$, we obtain

$$
\begin{aligned}
\frac{d}{d t} \Psi_{p}(\xi, t) & =\int_{0}^{t} \Upsilon_{p}^{\prime}(t-s)|\hat{u}(\xi, s)|^{2} d s+|\hat{u}(\xi, t)|^{2} \\
& \leq-p \int_{0}^{t}\left[\Upsilon_{p}(t-s)\right]^{q}|\hat{u}(\xi, s)|^{2} d s+\frac{1}{l|\xi|^{2}} \hat{E}(\xi, t) .
\end{aligned}
$$

Now, integrating the first inequality in (5.14) over $[0, \infty)$ and using the third inequality in the same formula (5.14), we obtain

$$
\begin{aligned}
\int_{0}^{\infty}|\hat{u}(\xi, s)|^{2} d s & \leq \frac{\hat{k}\left(1+|\xi|^{2}\right)}{2 l \varepsilon_{0}|\xi|^{4}} \hat{E}(\xi, 0) \\
& =\frac{c}{|\xi|^{2} \tilde{g}(\xi)} \hat{E}(\xi, 0) .
\end{aligned}
$$


Next, using Hölder's inequality and (5.16), we write

$$
\begin{aligned}
\int_{0}^{t}\left[\Upsilon_{p}(t-s)\right]^{q}|\hat{u}(\xi, s)|^{2} d s & \geq \frac{\left[\Psi_{p}(\xi, t)\right]^{q}}{\left(\int_{0}^{t}|\hat{u}(\xi, s)|^{2} d s\right)^{q-1}} \\
& \geq \frac{c\left(|\xi|^{2} \tilde{g}(\xi)\right)^{q-1}\left[\Psi_{p}(\xi, t)\right]^{q}}{[\hat{E}(\xi, 0)]^{q-1}} .
\end{aligned}
$$

Inserting (5.17) into (5.15), then the inequality (5.13) holds. This completes the proof of Lemma 5.3.

Proof of Theorem 5.2. The core of the proof relies on the following three steps.

Step 1. Here let us assume that $|\xi| \geq 1$, then following the same proof as in [1], we obtained

$$
\int_{|\xi| \geq 1} \hat{E}(\xi, t) d \xi \leq \frac{Q}{(1+t)^{p}}
$$

where $Q$ is the same positive constant defined in [1] and depending on $E(0),\left\|u_{0}\right\|_{2}$ and $\left\|u_{0}\right\|_{1}$.

Step 2. In this step, we assume that $|\xi|<1$, and we define the new functional $\tilde{\mathscr{L}}_{0}$ as

$$
\tilde{\mathscr{L}}_{0}(\xi, t)=\tilde{g}(\xi) \mathscr{L}(\xi, t)+\varepsilon|\xi|^{2} \tilde{g}(\xi) \Psi_{p}(\xi, t),
$$

where $\varepsilon$ is a small positive constant.

Taking the time derivative of (5.19), making use of the first inequality in (5.14) an (5.13) it holds that

$$
\frac{d}{d t} \tilde{\mathscr{L}}_{0}(\xi, t) \leq-2 \varepsilon_{0} \tilde{g}(\xi) \hat{E}(\xi, t)+\frac{\varepsilon \tilde{g}}{l} \hat{E}(\xi, t)-\frac{\varepsilon c}{[\hat{E}(\xi, 0)]^{q-1}}\left[|\xi|^{2} \tilde{g}(\xi) \Psi_{p}(\xi, t)\right]^{q} .
$$

Choosing $\varepsilon$ sufficiently small, we arrive at

$$
\frac{d}{d t} \tilde{\mathscr{L}}_{0}(\xi, t) \leq-\left(\varepsilon_{0} \tilde{g}(\xi) \hat{E}(\xi, t)+\frac{c}{[\hat{E}(\xi, 0)]^{q-1}}\left[|\xi|^{2} \tilde{g}(\xi) \Psi_{p}(\xi, t)\right]^{q}\right) .
$$

The second formula in (5.14) together with (5.19), ensure

$$
\frac{1}{\hat{k}} \hat{E}(\xi, t) \leq \tilde{\mathscr{L}}_{0}(\xi, t) \leq \hat{k} \hat{E}(\xi, t)+c|\xi|^{2} \tilde{g}(\xi) \Psi_{p}(\xi, t) .
$$

Also, the last formula in (5.14) and (5.19) imply

$$
\tilde{\mathscr{L}}_{0}(\xi, 0) \leq \hat{k} \hat{E}(\xi, 0) .
$$

Now, going back to (5.21), we also have that

$$
\begin{aligned}
{\left[\tilde{\mathscr{L}}_{0}(\xi, t)\right]^{q} } & \leq c[\hat{E}(\xi, t)]^{q}+c\left(\frac{\hat{E}(\xi, 0)}{\hat{E}(\xi, 0)}\right)^{q}\left[|\xi|^{2} \tilde{g}(\xi) \Psi_{p}(\xi, t)\right]^{q} \\
& \leq c \frac{[\hat{E}(\xi, 0)]^{q-1}}{\varepsilon_{0} \tilde{g}(\xi)}\left\{\varepsilon_{0} \tilde{g}(\xi) \hat{E}(\xi, t)+\frac{c}{[\hat{E}(\xi, 0)]^{q-1}}\left[|\xi|^{2} \tilde{g}(\xi) \Psi_{p}(\xi, t)\right]\right\}^{q} .
\end{aligned}
$$


Using (5.20), we deduce

$$
\frac{d}{d t} \tilde{\mathscr{L}}_{0}(\xi, t)+c \frac{\varepsilon_{0} \tilde{g}(\xi)}{[\hat{E}(\xi, 0)]^{q-1}}\left[\tilde{\mathscr{L}}_{0}(\xi, t)\right]^{q} \leq 0 .
$$

Applying Gronwall type inequality, we get

$$
\begin{aligned}
\tilde{\mathscr{L}}_{0}(\xi, t) & \leq\left\{\frac{t}{p} \frac{c \varepsilon_{0} \tilde{g}(\xi)}{[\hat{E}(\xi, 0)]^{q-1}}+\left[\tilde{\mathscr{L}}_{0}(\xi, t)\right]^{-(q-1)}\right\}^{-p} \\
& =\left\{\frac{c t}{p} \frac{|\xi|^{2} /\left(1+|\xi|^{2}\right)}{[\hat{E}(\xi, 0)]^{1 / p}}+\frac{1}{\left[\tilde{\mathscr{L}}_{0}(\xi, t)\right]^{1 / p}}\right\}^{-p} .
\end{aligned}
$$

Then, inequality (5.22) yields

$$
\begin{aligned}
\tilde{\mathscr{L}}_{0}(\xi, t) & \leq\left\{\frac{1}{[\hat{E}(\xi, 0)]^{1 / p}}\left\{\frac{t|\xi|^{2}}{1+|\xi|^{2}}\right\}+c\right\}^{-p} \\
& \leq \frac{c \hat{E}(\xi, 0)}{\left(1+\frac{t|\xi|^{2}}{1+|\xi|^{2}}\right)^{p}} .
\end{aligned}
$$

Since $|\xi|<1$, the last inequality implies

$$
\tilde{\mathscr{L}}_{0}(\xi, t) \leq \frac{c \hat{E}(\xi, 0)}{\left(1+\frac{t}{2}|\xi|^{2}\right)^{p}}
$$

Thus exploiting (5.21), we get

$$
\int_{|\xi|<1} \hat{E}(\xi, t) d \xi \leq c \int_{|\xi|<1} \frac{\hat{E}(\xi, 0)}{\left(1+\frac{t}{2}|\xi|^{2}\right)^{p}} d \xi .
$$

Step 3. Now, by using (5.12), we obtain from (5.24)

$$
\begin{aligned}
\int_{|\xi|<1} \frac{\hat{E}(\xi, 0)}{\left(1+\frac{t}{2}|\xi|^{2}\right)^{p}} \leq & C_{\gamma} \int_{|\xi|<1} \frac{1}{\left(1+\frac{t}{2}|\xi|^{2}\right)^{p}}\left(|\xi|^{2 \gamma+2}\left\|u_{0}\right\|_{1, \gamma}^{2}+|\xi|^{2 \gamma}\left\|u_{1}\right\|_{1, \gamma}^{2}\right) d \xi \\
& +C_{\gamma}\left|\int_{\mathbb{R}^{N}} u_{0}(x) d x\right|^{2} \int_{|\xi|<1} \frac{|\xi|^{2}}{\left(1+\frac{t}{2}|\xi|^{2}\right)^{p}} d \xi \\
& +C_{\gamma}\left|\int_{\mathbb{R}^{N}} u_{1}(x) d x\right| \int_{|\xi|<1} \frac{1}{\left(1+\frac{t}{2}|\xi|^{2}\right)^{p}} d \xi
\end{aligned}
$$

Using the fact that

$$
\frac{1}{\left(1+\frac{t}{2}|\xi|^{2}\right)^{p}} \leq \frac{C}{\left(1+t^{1 / 2}|\xi|\right)^{2 p}}, \text { for }|\xi|<1
$$


and passing to the polar coordinates, we get

$$
\begin{aligned}
\int_{|\xi|<1} \frac{\hat{E}(\xi, 0)}{\left(1+\frac{t}{2}|\xi| t^{2}\right)^{p}} \leq & C_{\gamma}\left\|u_{0}\right\|_{1, \gamma}^{2} \int_{0}^{1} \frac{r^{N-1+2 \gamma+2}}{\left(1+r t^{1 / 2}\right)^{2 p}} d r+C_{\gamma}\left\|u_{1}\right\|_{1, \gamma}^{2} \int_{0}^{1} \frac{r^{N-1+2 \gamma}}{\left(1+r t^{1 / 2}\right)^{2 p}} d r \\
& +C_{\gamma}\left|\int_{\mathbb{R}^{N}} u_{0}(x) d x\right|^{2} \int_{0}^{1} \frac{r^{N-1+2}}{\left(1+r t^{1 / 2}\right)^{2 p}} d r \\
& +C_{\gamma}\left|\int_{\mathbb{R}^{N}} u_{1}(x) d x\right|^{2} \int_{0}^{1} \frac{r^{N-1}}{\left(1+r t^{1 / 2}\right)^{2 p}} d r .
\end{aligned}
$$

Consequently, applying Lemma 2.2, we deduce that:

- If $2 p \neq\{N+2 \gamma+2, N+2 \gamma, N+2, N\}$, then we have

$$
\begin{aligned}
\int_{|\xi|<1} \hat{E}(\xi, t) d \xi \leq & C_{\gamma}\left\|u_{0}\right\|_{1, \gamma}^{2} t^{-\min (p,(N+2 \gamma+2) / 2)}+C_{\gamma}\left\|u_{1}\right\|_{1, \gamma}^{2} t^{-\min (p,(N+2 \gamma) / 2)} \\
& +C_{\gamma}\left|\int_{\mathbb{R}^{N}} u_{0}(x) d x\right|^{2} t^{-\min (p,(N+2) / 2)}+C_{\gamma}\left|\int_{\mathbb{R}^{N}} u_{1}(x) d x\right|^{2} t^{-\min (p, N / 2)} .
\end{aligned}
$$

- If $2 p=N+2 \gamma+2$, we get

$$
\begin{aligned}
\int_{|\xi|<1} \hat{E}(\xi, t) d \xi \leq & C_{\gamma}\left\|u_{0}\right\|_{1, \gamma}^{2} t^{-2 p} \log (2+t)+C_{\gamma}\left\|u_{1}\right\|_{1, \gamma}^{2} t^{-(N+2 \gamma) / 2} \\
& +C_{\gamma}\left|\int_{\mathbb{R}^{N}} u_{0}(x) d x\right|^{2} t^{-(N+2) / 2}+C_{\gamma}\left|\int_{\mathbb{R}^{N}} u_{1}(x) d x\right|^{2} t^{-N / 2} .
\end{aligned}
$$

- If $2 p=N+2 \gamma$, we have

$$
\begin{aligned}
\int_{|\xi|<1} \hat{E}(\xi, t) d \xi \leq & C_{\gamma}\left(\left\|u_{0}\right\|_{1, \gamma}^{2}+\left|\int_{\mathbb{R}^{N}} u_{0}(x) d x\right|^{2}\right) t^{-p}+C_{\gamma}\left\|u_{1}\right\|_{1, \gamma}^{2} t^{-p} \log (2+t) \\
& +C_{\gamma}\left|\int_{\mathbb{R}^{N}} u_{1}(x) d x\right|^{2} t^{-N / 2}
\end{aligned}
$$

- If $2 p=N+2$, we find

$$
\begin{aligned}
\int_{|\xi|<1} \hat{E}(\xi, t) d \xi \leq & C_{\gamma}\left\|u_{0}\right\|_{1, \gamma}^{2} t^{-p}+C_{\gamma}\left\|u_{1}\right\|_{1, \gamma}^{2} t^{-(N+2 \gamma) / 2}+C_{\gamma}\left|\int_{\mathbb{R}^{N}} u_{1}(x) d x\right|^{2} t^{-N / 2} \\
& +C_{\gamma}\left|\int_{\mathbb{R}^{N}} u_{0}(x) d x\right|^{2} t^{-(N+2) / 2} \log (2+t) .
\end{aligned}
$$

- If $2 p=N$, we obtain

$$
\begin{aligned}
\int_{|\xi|<1} \hat{E}(\xi, t) d \xi \leq & C_{\gamma}\left(\left\|u_{0}\right\|_{1, \gamma}^{2}+\left\|u_{1}\right\|_{1, \gamma}^{2}+\left|\int_{\mathbb{R}^{N}} u_{0}(x) d x\right|^{2}\right) t^{-2 p} \\
& +C_{\gamma}\left|\int_{\mathbb{R}^{N}} u_{1}(x) d x\right|^{2} t^{-p} \log (2+t)
\end{aligned}
$$


Now, by assuming that $\int_{\mathbb{R}^{N}} u_{i}(x) d x=0, i=0,1$, then the result of Theorem 5.2 follows from (5.18), (5.25), (5.26), (5.27), (5.28) and (5.29).

Remark 5.4 In Theorem 5.1, and Theorem 5.2 we could consider more general kernels as well, allowing $g$ to be flat, such that the set where $g^{\prime}=0$ is not too large (in a suitable sense) as in [21]. However, in this work, we will restrict to the decay rate of solutions, in order not to introduce further technical difficulties.

\section{Decay estimates for $f \neq 0$}

In this section, we consider problem (1.1)-(1.2) in the particular case $m=1, B^{j k}=\delta_{j k}$, and $K^{j k}(t)=\delta_{j k} g(t)$ with $f \neq 0$, where $\delta_{j k}$ is the Kronecker symbol. Namely, we are interested in the study of the following Cauchy problem

$$
\begin{cases}u_{t t}-\Delta u+\int_{0}^{t} g(t-s) \Delta u(s) d s+u_{t}+|u|^{p-1} u=0, & t>0, x \in \mathbb{R}^{N} \\ u(0, x)=u_{0}(x), u_{t}(0, x)=u_{1}(x), & x \in \mathbb{R}^{N}\end{cases}
$$

and we will discuss the global existence and the asymptotic behavior of the solution with the absorption $|u|^{p-1} u$ in both cases supercritical and subcritical. It is worthy to be mentioned that we do not require any compactness assumptions on the support of the initial data.

\subsection{The supercritical case $p>1+\frac{3}{N}$}

In the supercritical case, we will show that the solution will behave as that of the corresponding linear equation. We will use Duhamel's principle to express the solution to the nonhomogeneous problem $(f \neq 0)$ with the help of solution to the homogeneous problem $(f=0)$. We will show that the precise decay estimates to the linear equation (1.1) given in Theorem 3.1 play and essential role in the analysis of the nonlinear problem. Let us first define

$$
p_{0}(N)=1+\frac{3}{N}
$$

then we have:

Theorem 6.1 Let $p_{0}(N)<p<+\infty$ if $N=2$ or $p_{0}(N)<p \leq N /(N-2)$ if $N \geq 3$. Assume that $\left(u_{0}, u_{1}\right) \in$ $H^{1}\left(\mathbb{R}^{N}\right) \cap L^{1,1}\left(\mathbb{R}^{N}\right) \times L^{2}\left(\mathbb{R}^{N}\right) \cap L^{1,1}\left(\mathbb{R}^{N}\right)^{1}$ such that $\int_{\mathbb{R}^{N}} u_{i}=0, i=0,1$. Then there exists a positive number $\varepsilon>0$ such that if

$$
I_{0}^{2}=\int_{\mathbb{R}^{N}} e^{a|x|^{2} / 4}\left(\left|u_{1}(x)\right|^{2}+\left|\nabla u_{0}(x)\right|^{2}+\left|u_{0}(x)\right|^{2}+\left|u_{0}(x)\right|^{p}\right) d x<\varepsilon
$$

then problem (6.1) has a unique global solution u satisfying

$$
\left\|u_{t}(t)\right\|_{2}+\|\nabla u(t)\|_{2} \leq C(1+t)^{-N / 4-1 / 2}\left(\left\|u_{1}\right\|_{1,1}+\left\|u_{0}\right\|_{1,1}+\left\|u_{1}\right\|_{2}+\left\|\nabla u_{0}\right\|_{2}\right) .
$$

Remark 6.2 The existence of a global solution of problem (6.1) can be shown with the same method as in [14, Proposition 2.1] (see also [20, Proposition 2.1]). We omit it for its length.

\footnotetext{
${ }^{1}$ In fact these estimates hold for any $\gamma \in[0,1]$, so we take $\gamma=1$ because in this case we have fastes decay rate.
} 
Remark 6.3 If $\left(u_{0}, u_{1}\right) \in H^{1}\left(\mathbb{R}^{N}\right) \cap L^{1}\left(\mathbb{R}^{N}\right) \times L^{2}\left(\mathbb{R}^{N}\right) \cap L^{1}\left(\mathbb{R}^{N}\right)$, then we can prove the following decay estimate

$$
\left\|u_{t}(t)\right\|_{2}+\|\nabla u(t)\|_{2} \leq C(1+t)^{-N / 4}\left(\left\|u_{1}\right\|_{1}+\left\|u_{0}\right\|_{1}+\left\|u_{1}\right\|_{2}+\left\|\nabla u_{0}\right\|_{2}\right)
$$

provided that (6.2) holds and $p_{0}(N)=1+3 /(N-1)$. Theorem 6.1 shows that by taking the initial data $\left(u_{0}, u_{1}\right) \in H^{1}\left(\mathbb{R}^{N}\right) \cap L^{1,1}\left(\mathbb{R}^{N}\right) \times L^{2}\left(\mathbb{R}^{N}\right) \cap L^{1,1}\left(\mathbb{R}^{N}\right)$ satisfying $\int_{\mathbb{R}^{N}} u_{i}=0, i=0,1$, then we can shift the critical power $p_{0}$ to be $p_{0}(N)=1+\frac{3}{N}$, and the decay rate can be improved by $t^{-1 / 2}$.

Remark 6.4 Theorem 6.1 has been proved without assuming any compactness assumptions of the support on the initial data. Recently, Ikehata and Tanizawa [14] also removed the compactness assumption for the damped wave equation by using a weight function similar to the one defined in (6.4).

The basic idea in our proof of Theorem 6.1 is based on the result of Theorem 3.1 and the weighted energy estimate used in [27] and [14].

As in [14] (see also [27]), we define the function

$$
\phi(t, x)=\frac{a|x|^{2}}{4(t+1)}, \quad a>0,
$$

as a weight function satisfying the following properties

$$
\left\{\begin{array}{l}
\phi_{t}(t, x)<0, \\
a \phi_{t}(t, x)+|\nabla \phi(t, x)|^{2}=0, \\
|\nabla \phi(t, x)|=\frac{a x}{2(t+1)},|\nabla \phi(t, x)|^{2}=\frac{a^{2}|x|^{2}}{4(t+1)^{2}} .
\end{array}\right.
$$

For $v>0$ and $t \geq 0$, we define a family of weighted function spaces $H_{v \phi(t, .)}^{1}\left(\mathbb{R}^{N}\right)$ as:

$$
f \in H_{v \phi(t, .)}^{1}\left(\mathbb{R}^{N}\right) \Leftrightarrow f \in H^{1}\left(\mathbb{R}^{N}\right),\left\|e^{v \phi(t, .)} f\right\|_{2}^{2}+\left\|e^{v \phi(t, .)} \nabla f\right\|_{2}^{2}<+\infty, \forall t \geq 0 .
$$

We recall the Gagliardo-Nirenberg type inequality introduced in [14].

Lemma 6.5 Let $\theta(q)=N(1 / 2-1 / q)$ and $0 \leq \theta(q) \leq 1$ and $0 \leq v \leq 1$. If $v \in H_{v \phi(t, .)}^{1}\left(\mathbb{R}^{N}\right)$ then

$$
\left\|e^{v \phi(t, .)} v\right\|_{q} \leq C_{v}(1+t)^{(1-\theta(q)) / 2}\|\nabla v\|_{2}^{1-v}\left\|e^{\phi(t, .)} \nabla v\right\|_{2}^{v}
$$

with some constant $C_{v}>0$.

Then, the following result holds: (see [14, Lemma 2.5])

Lemma 6.6 For each $\delta>0$, there exists a constant $C=C_{\delta, p}$ such that

$$
\int_{\mathbb{R}^{N}} e^{-2 p \delta \phi(t, x)} d x \leq C(1+t)^{N / 2}
$$

The following lemma is crucial in our argument.

Lemma 6.7 There exists a constant $C=C_{\delta}$ such that for all $t \geq 0$ we have

$$
\int_{\mathbb{R}^{N}} e^{-2 p \delta \phi(t, x)}(1+|x|)^{2} d x \leq C(1+t)^{(N+2) / 2} .
$$


Proof. It follows from (6.4) that

$$
\begin{aligned}
\int_{\mathbb{R}^{N}} e^{-2 p \delta \phi(t, x)}(1+|x|)^{2} d x & =\int_{\mathbb{R}^{N}} e^{-\frac{p a \delta|x|^{2}}{(t+1)}}(1+|x|)^{2} d x \\
& \leq 4 \int_{|x| \leq 1} e^{-\frac{p a \delta \mid x x^{2}}{(t+1)}}+4 \int_{|x| \geq 1} e^{-\frac{p a \delta|x|^{2}}{(t+1)}}|x|^{2} d x \\
& \leq 4 c_{1}+4 \tilde{K}_{1} .
\end{aligned}
$$

Concerning the integral $\tilde{K}_{1}$, we make the change of variable $y=x / \sqrt{t+1}$, then we get $d y=d x /(\sqrt{t+1})^{N}$, which gives

$$
\begin{aligned}
\tilde{K}_{1} & =\int_{|y| \geq \frac{1}{\sqrt{t+1}}} e^{-p a \delta|y|^{2}}|y|^{2}(1+t)^{\frac{N+2}{2}} d y \\
& \leq(1+t)^{\frac{N+2}{2}} \int_{\mathbb{R}^{N}} e^{-p a \delta|y|^{2}}|y|^{2} d y \\
& \leq c_{2}(1+t)^{\frac{N+2}{2}} .
\end{aligned}
$$

This concludes the proof of Lemma 6.7.

Now, let us define

$$
\mathscr{E}(t)=e^{2 \phi}\left\{\frac{1}{2} u_{t}^{2}+\frac{1}{2}\left(1-\int_{0}^{t} g(s) d s\right)|\nabla u|^{2}+\frac{1}{2}(g \diamond \nabla u)(t)+\frac{1}{p+1}|u|^{p+1}\right\},
$$

then we have:

Lemma 6.8 Let $u$ be a solution of (6.1), then we have, for any $\eta>0$,

$$
\begin{aligned}
\frac{d}{d t} \mathscr{E}(t) \leq & 2 \frac{\phi_{t}}{p+1} e^{2 \phi}|u|^{p+1}+\operatorname{div}\left(e^{2 \phi} u_{t}\left(\nabla u-\int_{0}^{t} g(t-s) \nabla u(s) d s\right)\right) \\
& +\frac{e^{2 \phi}}{l \phi_{t}}\left(l \phi_{t} \nabla u-u_{t} \nabla \phi\right)^{2}-e^{2 \phi}\left\{\left(1-a\left(\frac{1-l}{l}\right)-a\right)+\phi_{t}\left(\frac{a}{2 \mu_{1}}-1\right)\right\} u_{t}^{2} \\
& -\frac{e^{2 \phi}}{2} g(t)|\nabla u|^{2}-\left(\zeta-\frac{1}{2}\left(1+\frac{1}{\eta}\right)(1-l) \mu_{1}\right) e^{2 \phi}(g \diamond \nabla u)(t) \\
& +\phi_{t} e^{2 \phi}(g \diamond \nabla u)(t)+\frac{\mu_{1}}{2} e^{2 \phi}(1+\eta)(1-l)^{2}|\nabla u(t)|^{2} d x .
\end{aligned}
$$

Proof. Multiplying equation (6.1) by $e^{2 \phi} u_{t}$, and using (6.5), we obtain

$$
\begin{aligned}
0= & \frac{d}{d t}\left(\frac{e^{2 \phi}}{2}\left(u_{t}^{2}+|\nabla u|^{2}\right)+\frac{e^{2 \phi}}{p+1}|u|^{p+1}\right) \\
& -\operatorname{div}\left(e^{2 \phi} u_{t} \nabla u\right)+e^{2 \phi}\left(\left(1+\frac{|\nabla \phi|^{2}}{\phi_{t}}\right)-\phi_{t}\right) u_{t}^{2} \\
& -\frac{e^{2 \phi}}{\phi_{t}}\left(\phi_{t} \nabla u-u_{t} \nabla \phi\right)^{2}-2 \frac{\phi_{t}}{p+1} e^{2 \phi}|u|^{p+1} \\
& +e^{2 \phi} u_{t} \int_{0}^{t} g(t-s) \Delta u(s) d s .
\end{aligned}
$$


Lemma 2.1 implies

$$
\begin{aligned}
e^{2 \phi} u_{t} \int_{0}^{t} g(t-s) \Delta u(s) d s= & \frac{e^{2 \phi}}{2} g(t)|\nabla u|^{2}-\frac{e^{2 \phi}}{2}\left(g^{\prime} \diamond \nabla u\right)(t) \\
& +\operatorname{div}\left(e^{2 \phi} u_{t} \int_{0}^{t} g(t-s) \nabla u(s) d s\right) \\
& +\frac{d}{d t}\left[\frac{e^{2 \phi}}{2}\left((g \diamond \nabla u)(t)-\left(\int_{0}^{t} g(s) d s\right)|\nabla u(t)|^{2}\right)\right] \\
& -\phi_{t} e^{2 \phi}\left[(g \diamond \nabla u)(t)-\left(\int_{0}^{t} g(s) d s\right)|\nabla u(t)|^{2}\right] \\
& -2 \nabla \phi e^{2 \phi}\left[u_{t} \int_{0}^{t} g(t-s) \nabla u(s) d s\right] .
\end{aligned}
$$

Then, from (6.10) and (6.11), we obtain

$$
\begin{aligned}
2 \frac{\phi_{t}}{p+1} e^{2 \phi}|u|^{p+1}= & \frac{d}{d t} \mathscr{E}(t)-\operatorname{div}\left(e^{2 \phi} u_{t}\left(\nabla u-\int_{0}^{t} g(t-s) \nabla u(s) d s\right)\right) \\
& -\frac{e^{2 \phi}}{\phi_{t}}\left(\phi_{t} \nabla u-u_{t} \nabla \phi\right)^{2}+e^{2 \phi}\left(\left(1+\frac{|\nabla \phi|^{2}}{\phi_{t}}\right)-\phi_{t}\right) u_{t}^{2} \\
& +\frac{e^{2 \phi}}{2} g(t)|\nabla u|^{2}-\frac{e^{2 \phi}}{2}\left(g^{\prime} \diamond \nabla u\right)(t) \\
& -\phi_{t} e^{2 \phi}\left[(g \diamond \nabla u)(t)-\left(\int_{0}^{t} g(s) d s\right)|\nabla u(t)|^{2}\right] \\
& -2 \nabla \phi e^{2 \phi}\left[u_{t} \int_{0}^{t} g(t-s) \nabla u(s) d s\right]
\end{aligned}
$$

Now, the last term in (6.12) can be estimated as follows: $\forall \mu_{1}>0$,

$$
\begin{aligned}
& \left|-2 \nabla \phi e^{2 \phi}\left(u_{t} \int_{0}^{t} g(t-s) \nabla u(s) d s\right)\right| \\
\leq & \frac{1}{2 \mu_{1}} e^{2 \phi}|\nabla \phi|^{2} u_{t}^{2}+\frac{\mu_{1}}{2} e^{2 \phi}\left(\int_{0}^{t} g(t-s) \nabla u(s) d s\right)^{2} .
\end{aligned}
$$

We have, for any $\eta>0$,

$$
\begin{aligned}
\left(\int_{0}^{t} g(t-s) \nabla u(s) d s\right)^{2} \leq & \left(\int_{0}^{t} g(t-s)|\nabla u(s)-\nabla u(t)|+|\nabla u(t)| d s\right)^{2} \\
\leq & (1+\eta)\left(\int_{0}^{t} g(t-s)|\nabla u(t)| d s\right)^{2} \\
& +\left(1+\frac{1}{\eta}\right)\left(\int_{0}^{t} g(t-s)|\nabla u(s)-\nabla u(t)| d s\right)^{2} \\
\leq & \left(1+\frac{1}{\eta}\right)(1-l)(g \diamond \nabla u)(t)+(1+\eta)(1-l)^{2}|\nabla u(t)|^{2}
\end{aligned}
$$


Consequently, inequality (6.13) takes the form

$$
\begin{aligned}
& \left|-2 \nabla \phi e^{2 \phi}\left(u_{t} \int_{0}^{t} g(t-s) \nabla u(s) d s\right)\right| \\
\leq & \frac{1}{2 \mu_{1}} e^{2 \phi}|\nabla \phi|^{2} u_{t}^{2}+\frac{\mu_{1}}{2} e^{2 \phi}\left(1+\frac{1}{\eta}\right)(1-l)(g \diamond \nabla u)(t) \\
& +\frac{\mu_{1}}{2} e^{2 \phi}(1+\eta)(1-l)^{2}|\nabla u(t)|^{2} d x .
\end{aligned}
$$

Since our kernel $g$ satisfying (2.2) and by using the fact that $|\nabla \phi|^{2}=-a \phi_{t}$, we find from (6.12) and (6.15)

$$
\begin{aligned}
\frac{d}{d t} \mathscr{E}(t) \leq & 2 \frac{\phi_{t}}{p+1} e^{2 \phi}|u|^{p+1}+\operatorname{div}\left(e^{2 \phi} u_{t}\left(\nabla u-\int_{0}^{t} g(t-s) \nabla u(s) d s\right)\right) \\
& +\frac{e^{2 \phi}}{\phi_{t}}\left(\phi_{t} \nabla u-u_{t} \nabla \phi\right)^{2}-e^{2 \phi}\left(\left(1+\frac{|\nabla \phi|^{2}}{\phi_{t}}\right)+\phi_{t}\left(\frac{a}{2 \mu_{1}}-1\right)\right) u_{t}^{2} \\
& -\frac{e^{2 \phi}}{2} g(t)|\nabla u|^{2}-\left(\zeta-\frac{1}{2}\left(1+\frac{1}{\eta}\right)(1-l) \mu_{1}\right) e^{2 \phi}(g \diamond \nabla u)(t) \\
& +\phi_{t} e^{2 \phi}\left[(g \diamond \nabla u)(t)-(1-l)|\nabla u(t)|^{2}\right] \\
& +\frac{\mu_{1}}{2} e^{2 \phi}(1+\eta)(1-l)^{2}|\nabla u(t)|^{2} d x
\end{aligned}
$$

where we have used also the inequality

$$
-\phi_{t}\left(\int_{0}^{t} g(s) d s\right) \leq-\phi_{t}\left(\int_{0}^{\infty} g(s) d s\right)=-\phi_{t}(1-l) .
$$

Observing,

$$
\begin{aligned}
& \frac{e^{2 \phi}}{\phi_{t}}\left(\phi_{t} \nabla u-u_{t} \nabla \phi\right)^{2}-\phi_{t} e^{2 \phi}(1-l)|\nabla u|^{2} \\
= & \frac{e^{2 \phi}}{l \phi_{t}}\left(l \phi_{t} \nabla u-u_{t} \nabla \phi\right)^{2}-\left(\frac{1-l}{l}\right) e^{2 \phi} \frac{u_{t}^{2}|\nabla \phi|^{2}}{\phi_{t}} \\
= & \frac{e^{2 \phi}}{l \phi_{t}}\left(l \phi_{t} \nabla u-u_{t} \nabla \phi\right)^{2}+a\left(\frac{1-l}{l}\right) e^{2 \phi} u_{t}^{2} .
\end{aligned}
$$

Since $\frac{|\nabla \phi|^{2}}{\phi_{t}}=-a,(6.9)$ holds.

Next, let us define

$$
\mathscr{H}(t)=e^{2 \phi}\left(u_{t} u+\frac{1}{2} u^{2}\right) .
$$

Then we have the following estimate. 
Lemma 6.9 Let $u$ be a solution of (6.1), then we have

$$
\begin{aligned}
& \frac{d}{d t} \mathscr{H}(t)+e^{2 \phi}\left(\left(\frac{l}{2}-2 a-\frac{\mu_{2}(1-l)}{2}\right)|\nabla u|^{2}+\left(\frac{1}{4 a}-\frac{1}{2 \mu_{2}}\right)|\nabla \phi|^{2} u^{2}\right) \\
& -e^{2 \phi}\left(1+\frac{4}{a}|\nabla \phi|^{2}\right) u_{t}^{2}+e^{2 \phi}|u|^{p+1} \\
\leq & e^{2 \phi}\left(\frac{1-l}{2 l}\left(1+\mu_{2}\right)\right)(g \diamond \nabla u)(t) \\
& +\operatorname{div}\left[e^{2 \phi}\left(u(t) \int_{0}^{t} g(t-s) \nabla u(s) d s\right)-\nabla u u\right] .
\end{aligned}
$$

Proof. Multiplying equation (6.1) by $e^{2 \phi} u$ we obtain

$$
\begin{aligned}
& \frac{d}{d t} e^{2 \phi}\left(u_{t} u+\frac{1}{2} u^{2}\right)+e^{2 \phi}\left(|\nabla u|^{2}+\frac{1}{a}|\nabla \phi|^{2} u^{2}\right)-e^{2 \phi} u_{t}^{2} \\
\leq & e^{2 \phi}\left[\frac{2}{a}|\nabla \phi|^{2}\left|u_{t} u\right|+2|\nabla \phi||\nabla u||u|\right]+e^{2 \phi}\left|\nabla u(t) \int_{0}^{t} g(t-s) \nabla u(s) d s\right| \\
& +e^{2 \phi}\left(2|\nabla \phi|\left|u(t) \int_{0}^{t} g(t-s) \nabla u(s) d s\right|\right) \\
& +\frac{d}{d x}\left[e^{2 \phi}\left(u(t) \int_{0}^{t} g(t-s) \nabla u(s) d s\right)-\nabla u \cdot u\right]+e^{2 \phi}|u|^{p+1} .
\end{aligned}
$$

Now, we have, for any $\eta>0$,

$$
\begin{aligned}
\left|\nabla u(t) \int_{0}^{t} g(t-s) \nabla u(s) d s\right| \leq & \frac{1}{2}\left(1+(1+\eta)(1-l)^{2}\right)|\nabla u(t)|^{2} \\
& +\frac{1}{2}\left(1+\frac{1}{\eta}\right)(1-l)(g \diamond \nabla u)(t) .
\end{aligned}
$$

Using (6.14), and Young's inequality, we have, for any $\mu_{2}>0$,

$$
\begin{aligned}
& \left(2|\nabla \phi|\left|u(t) \int_{0}^{t} g(t-s) \nabla u(s) d s\right|\right) \\
\leq & \frac{1}{2 \mu_{2}}|\nabla \phi|^{2} u^{2}+\frac{\mu_{2}}{2}\left(1+\frac{1}{\eta}\right)(1-l)(g \diamond \nabla u)(t) \\
& +\frac{\mu_{2}}{2}(1+\eta)(1-l)^{2}|\nabla u(t)|^{2} d x .
\end{aligned}
$$

By choosing $\eta=l /(1-l)$ in (6.21) and (6.22), we arrive at

$$
\left|\nabla u(t) \int_{0}^{t} g(t-s) \nabla u(s) d s\right| \leq\left(1-\frac{l}{2}\right)|\nabla u(t)|^{2}+\frac{1}{2} \frac{(1-l)}{l}(g \diamond \nabla u)(t)
$$

and

$$
\begin{aligned}
\left(2|\nabla \phi|\left|u(t) \int_{0}^{t} g(t-s) \nabla u(s) d s\right|\right) \leq & \frac{1}{2 \mu_{2}}|\nabla \phi|^{2} u^{2}+\frac{\mu_{2}}{2} \frac{(1-l)}{l}(g \diamond \nabla u)(t) \\
& +\frac{\mu_{2}}{2}(1-l)|\nabla u(t)|^{2} .
\end{aligned}
$$


Also, the first term in the right hand side of (6.20) can be estimated as follows:

$$
\frac{2}{a}|\nabla \phi|^{2}\left|u_{t} u\right|+2|\nabla \phi||\nabla u||u| \leq \frac{4}{a}|\nabla \phi|^{2} u_{t}^{2}+2 a|\nabla u|^{2}+\frac{3}{4 a}|\nabla \phi|^{2} u^{2} .
$$

Inserting the above estimates into (6.20), we obtain (6.19).

Now, for $\varepsilon$ small enough to be fixed later, we define the function $\mathscr{L}(t)$ as

$$
\mathscr{L}(t):=\mathscr{E}(t)+\varepsilon \mathscr{H}(t) .
$$

Then, we have the following estimate.

Lemma 6.10 Let $u$ be a solution of (6.1), then we have

$$
\int_{\mathbb{R}^{N}} \mathscr{L}(t) d x \leq \int_{\mathbb{R}^{N}} \mathscr{L}(0) d x+2 \int_{0}^{t} \int_{\mathbb{R}^{N}} \frac{\phi_{t}}{p+1} e^{2 \phi}|u|^{p+1} d x, \quad \forall t>0 .
$$

Proof. By using (6.9), (6.19) and (6.26), we obtain

$$
\begin{aligned}
\frac{d}{d t} \mathscr{L}(t) \leq & 2 \frac{\phi_{t}}{p+1} e^{2 \phi}|u|^{p+1}+\operatorname{div}\left(e^{2 \phi} u_{t}\left(\nabla u-\int_{0}^{t} g(t-s) \nabla u(s) d s\right)\right) \\
& +\frac{e^{2 \phi}}{l \phi_{t}}\left(l \phi_{t} \nabla u-u_{t} \nabla \phi\right)^{2}-\varepsilon\left(\frac{1}{4 a}-\frac{1}{2 \mu_{2}}\right)|\nabla \phi|^{2} u^{2} \\
& -e^{2 \phi}\left\{\left(1-a\left(\frac{1-l}{l}\right)-a\right)+\phi_{t}\left(\frac{a}{2 \mu_{1}}-1\right)-\varepsilon\left(1+\frac{4}{a}|\nabla \phi|^{2}\right)\right\} u_{t}^{2} \\
& -\frac{e^{2 \phi}}{2} g(t)|\nabla u|^{2}-\left(\zeta-\varepsilon\left(\frac{1-l}{2 l}\right)\left(1+\mu_{2}\right)-\frac{1}{2}\left(1+\frac{1}{\eta}\right)(1-l) \mu_{1}\right) e^{2 \phi}(g \diamond \nabla u)(t) \\
& +\phi_{t} e^{2 \phi}(g \diamond \nabla u)(t)+e^{2 \phi}\left(\frac{\mu_{1}}{2}(1+\eta)(1-l)^{2}-\varepsilon\left(\frac{l}{2}-2 a-\frac{\mu_{2}}{2}\right)\right)|\nabla u(t)|^{2} d x \\
& +\varepsilon d i v\left[e^{2 \phi}\left(u(t) \int_{0}^{t} g(t-s) \nabla u(s) d s\right)-\nabla u \cdot u\right]-\varepsilon e^{2 \phi}|u|^{p+1}
\end{aligned}
$$

Our goal now is to choose the constants $a, \varepsilon, \mu_{1}, \mu_{2}$ in (6.28). First, let us take $\eta=l /(1-l), \mu_{2}=2 a$ and $\mu_{1}=a$ and after that we write $\phi_{t}=-\frac{1}{a}|\nabla \phi|^{2}$, and fix $\varepsilon$ small such that

$$
\varepsilon<\min \left(1 / 8, \frac{\zeta l}{1-l}\right) \text {. }
$$

Then, we take $a$ small enough such that

$$
a<\min \left(l / 12, \frac{\varepsilon l}{2(1-l)}, \frac{\zeta l}{(1-l)(2 \varepsilon+1)}\right) .
$$

Therefore, (6.28) takes the form

$$
\begin{aligned}
\frac{d}{d t} \mathscr{L}(t) \leq & 2 \frac{\phi_{t}}{p+1} e^{2 \phi}|u|^{p+1}+\operatorname{div}\left(e^{2 \phi} u_{t}\left(\nabla u-\int_{0}^{t} g(t-s) \nabla u(s) d s\right)\right) \\
& \varepsilon \operatorname{div}\left[e^{2 \phi}\left(u(t) \int_{0}^{t} g(t-s) \nabla u(s) d s\right)-\nabla u . u\right] .
\end{aligned}
$$

Integrating the above inequality over $(0, t) \times \mathbb{R}^{N}$, then (6.27) holds. 
Lemma 6.11 Let $u$ be a solution of (6.1), then we have

$$
\begin{aligned}
& \int_{\mathbb{R}^{N}} e^{2 \phi}\left(\left|u_{t}\right|^{2}+|\nabla u|^{2}+|u|^{2}+(g \diamond \nabla u)(t)+|u|^{p+1}\right) d x \\
\leq & C I_{0}^{2}+C\left(\sup _{[0, t]}(1+s)^{\delta}\left\|e^{\gamma \phi(s, .)} u(s, .)\right\|_{p+1}\right)^{p+1}
\end{aligned}
$$

where $1 \geq \gamma>2 /(p+1), \delta>0$ and $C=C_{\delta, \gamma}>0$ is a constant, which depends on $\delta$ and $\gamma$.

Proof. By using Young's inequality, it is easy to see that from (6.8), (6.18) and (6.26) there exist two positive constants $\beta_{1}$ and $\beta_{2}$ depending on $\varepsilon$ such that

$$
\begin{aligned}
& \beta_{1} e^{2 \phi}\left(\left|u_{t}\right|^{2}+|\nabla u|^{2}+|u|^{2}+(g \diamond \nabla u)(t)+|u|^{p+1}\right) \\
\leq & \mathscr{L}(t) \leq \beta_{2} e^{2 \phi}\left(\left|u_{t}\right|^{2}+|\nabla u|^{2}+|u|^{2}+(g \diamond \nabla u)(t)+|u|^{p+1}\right) .
\end{aligned}
$$

From (6.27) and (6.31), we arrive at

$$
\begin{aligned}
& \int_{\mathbb{R}^{N}} e^{2 \phi}\left(\left|u_{t}\right|^{2}+|\nabla u|^{2}+|u|^{2}+(g \diamond \nabla u)(t)+|u|^{p+1}\right) d x \\
\leq & C \int_{\mathbb{R}^{N}} e^{2 \phi(0, x)}\left(\left|u_{1}\right|^{2}+\left|\nabla u_{0}\right|^{2}+\left|u_{0}\right|^{2}+\left|u_{0}\right|^{p+1}\right) d x \\
& +2 \int_{0}^{t} \int_{\mathbb{R}^{N}} \frac{\phi_{s}}{p+1} e^{2 \phi}|u|^{p+1} d x d s \\
\leq & C I_{0}^{2}+C \int_{0}^{t} \int_{\mathbb{R}^{N}} \frac{\left|\phi_{s}\right|}{p+1} e^{2 \phi}|u|^{p+1} d x d s \\
\leq & C I_{0}^{2}+C \int_{0}^{t}\left(\max _{x \in \mathbb{R}^{N}} \bar{\phi}(s, x)\right)\left\|e^{\gamma \phi(s, .)} u(s, .)\right\|_{p+1}^{p+1} d s
\end{aligned}
$$

where

$$
\bar{\phi}(s, x)=\left|\phi_{s}(s, x)\right| e^{(2-\gamma(p+1)) \phi(s, x)},
$$

with $\gamma$ such that

$$
\gamma>\frac{2}{p+1} .
$$

Following the same method as in [14], we can show that

$$
\max _{x \in \mathbb{R}^{N}} \bar{\phi}(s, x) \leq \frac{C_{\gamma}}{1+s}
$$

with some constant $C_{\gamma}>0$ for any $\gamma$ satisfying (6.33). Now, the proof of Lemma 6.11 can be finished along the same line as in [14]. Indeed, (6.32) implies

$$
\begin{aligned}
& \int_{\mathbb{R}^{N}} e^{2 \phi}\left(\left|u_{t}\right|^{2}+|\nabla u|^{2}+|u|^{2}+(g \diamond \nabla u)(t)+|u|^{p+1}\right) d x \\
\leq & C I_{0}^{2}+C_{\gamma} \int_{0}^{t} \frac{1}{1+s}\left\|e^{\gamma \phi(s, .)} u(s, .)\right\|_{p+1}^{p+1} d s \\
\leq & C I_{0}^{2}+C_{\gamma} \int_{0}^{t} \frac{1}{(1+s)^{1+\delta(p+1)}}\left\{\sup _{[0, t]}(1+s)^{\delta}\left\|e^{\gamma \phi(s, .)} u(s, .)\right\|_{p+1}\right\}^{p+1} d s,
\end{aligned}
$$


and since

$$
\int_{0}^{\infty} \frac{1}{(1+s)^{1+\delta(p+1)}} d s=C_{\delta}<+\infty,
$$

then (6.35) implies (6.30). This completes the proof of Lemma 6.11.

\section{Proof of Theorem 6.1}

Let $S(t) g$ denotes the unique solution of the Cauchy problem (1.2), (1.3) with $f=0, u_{0}=0$ and $g=u_{1}$. Then the solution $u$ of the nonlinear problem (1.2), (1.3) (with $f \neq 0$ ) can be represented as follows

$$
u(t)=S(t)\left(u_{0}+u_{1}\right)+\partial_{t}\left(S(t) u_{0}\right)-\int_{0}^{t} S(t-s)|u(s)|^{p-1} u(s) d s .
$$

We denote by $u_{L}(t)=S(t)\left(u_{0}+u_{1}\right)+\partial_{t}\left(S(t) u_{0}\right)$ the solution of the linear equation (i.e. $f=0$ ) with the initial data $\left.u_{L}\right|_{t=0}=u_{0}$ and $\left.\partial_{t} u_{L}\right|_{t=0}=u_{1}$ and

$$
u_{N}(t)=-\int_{0}^{t} S(t-s)|u(s)|^{p-1} u(s) d s .
$$

Let $D=\left(\partial_{t}, \partial_{x}\right)$, then from Theorem 3.1, we deduce, for $k=0$, the following estimate

$$
\left\|D u_{L}(t)\right\|_{2} \leq C(1+t)^{-N / 4-1 / 2}\left(\left\|u_{1}\right\|_{1,1}+\left\|u_{0}\right\|_{1,1}+\left\|u_{1}\right\|_{2}+\left\|\nabla u_{0}\right\|_{2}\right),
$$

where from now on we will denote by $C$ various positive constants which may be different at different occurrences. Our goal now is to estimate the nonlinear term $u_{N}(t)$ defined in (6.37). Indeed, applying the result of Theorem 3.1 with $\gamma=1$, we have for the first integral in (6.37)

$$
\left\|D S(t-s)|u(s)|^{p-1} u(s)\right\|_{2} \leq C(1+t-s)^{-N / 4-1 / 2}\left(\|u(s)\|_{2 p}^{p}+\|u(s)\|_{p, 1}^{p}\right) .
$$

To estimate the term $\|u(s)\|_{p, 1}^{p}$, we have from the Schwarz inequality and (6.7)

$$
\begin{aligned}
\|u(s)\|_{p, 1}^{p} & =\int_{\mathbb{R}^{N}} e^{-p \delta \phi(s, x)}|u(s, x)|^{p}(1+|x|) e^{p \delta \phi(s, x)} d x \\
& \leq\left(\int_{\mathbb{R}^{N}} e^{2 p \delta \phi(s, x)}|u(s, x)|^{2 p}\right)^{1 / 2}\left(\int_{\mathbb{R}^{N}} e^{-2 p \delta \phi(s, x)}(1+|x|)^{2}\right)^{1 / 2} \\
& \leq C(1+t)^{(N+2) / 4}\left\|e^{\delta \phi(s, x)} u(s)\right\|_{2 p}^{p} .
\end{aligned}
$$

On the other hand, by using [14, Lemma 2.6], we may estimate the norm $\|u(s)\|_{2 p}^{p}$ as

$$
\|u(s)\|_{2 p}^{p} \leq C_{\delta}(1+s)^{N / 4}\left\|e^{\delta \phi(s, x)} u(s)\right\|_{2 p}^{p} .
$$

By combining (6.39), (6.40) and (6.41), we find

$$
\left\|D S(t-s)|u(s)|^{p-1} u(s)\right\|_{2} \leq C(1+t-s)^{-N / 4-1 / 2}(1+t)^{(N+2) / 4}\left\|e^{\delta \phi(s, x)} u(s)\right\|_{2 p}^{p} .
$$

Now, to estimate the term $u_{N}(t)$, we follow the same method as in [27] and [14]. Indeed, we have for any $\varepsilon>0$

$$
\left\|D u_{N}(t)\right\|_{2} d s \leq C(1+t)^{-N / 4-1 / 2}\left(\sup _{[0, t]}(1+s)^{\beta}\left\|e^{\delta \phi(s, x)} u(s)\right\|_{2 p}\right)^{p}
$$


where

$$
\beta=\frac{N+6}{4 p}+\frac{\varepsilon}{p}
$$

From (6.38), and (6.43), we obtain

$$
(1+t)^{N / 4+1 / 2}\|D u(t)\|_{2} \leq C\left\{I_{0}+\left(\sup _{[0, t]}(1+s)^{\beta}\left\|e^{\delta \phi(s, x)} u(s)\right\|_{2 p}\right)^{p}\right\}
$$

Let us now define the functional

$$
W(t)=\left\|e^{\phi(t, x)} D u(t)\right\|_{2}+(1+t)^{N / 4+1 / 2}\|D u(t)\|_{2} .
$$

From Lemma 6.11, we deduce

$$
\left\|e^{\phi(t, x)} D u(t)\right\|_{2} \leq C I_{0}+C\left(\sup _{[0, t]}(1+s)^{\delta}\left\|e^{\gamma \phi(s, .)} u(s, .)\right\|_{p+1}\right)^{(p+1) / 2} .
$$

It follows from (6.45) and (6.47) that

$$
\begin{aligned}
W(t) \leq & C I_{0}+C\left(\sup _{[0, t]}(1+s)^{\beta}\left\|e^{\delta \phi(t, x)} u(s)\right\|_{2 p}\right)^{p} \\
& +C\left(\sup _{[0, t]}(1+s)^{\delta}\left\|e^{\gamma \phi(s, .)} u(s, .)\right\|_{p+1}\right)^{(p+1) / 2}
\end{aligned}
$$

for any $\gamma$ satisfying (6.33) and any $\delta>0$.

Applying Lemma 6.5 for $q=2 p$ and $q=p+1$, we get (see [14])

$$
\left\{\begin{array}{l}
\left\|e^{\delta \phi(t, x)} u(s)\right\|_{2 p} \leq C(1+s)^{(1-\theta(2 p)) / 2-(N+2)(1-\delta) / 4} W(s), \\
\left\|e^{\gamma \phi(s, .)} u(s, .)\right\|_{p+1} \leq C(1+s)^{(1-\theta(p+1)) / 2-(N+2)(1-\gamma) / 4} W(s) .
\end{array}\right.
$$

From (6.33), we can choose $\gamma$ as $\gamma=2 /(p+1)+\varepsilon_{1}$. Now, by the definition of $\theta(2 p)$ and $\theta(p+1)$ in Lemma 6.5 and, if we pick $\varepsilon, \delta$ and $\varepsilon_{1}$ are small enough we get

$$
\beta+(1-\theta(2 p)) / 2-(N+2)(1-\delta) / 4<0
$$

for $p>1+3 / N$ and

$$
\delta+(1-\theta(p+1)) / 2-(N+2)(1-\gamma) / 4<0
$$

for $p>1+2 / N$.

Consequently, the remaining part of the proof can be finished, following the same steps as in [27] or [14]. We give the details for the reader's convenience. Let the parameters as above be fixed, then we have from (6.48) and (6.49)

$$
\sup _{[0, t]} W(s) \leq C I_{0}+C\left(\sup _{[0, t]} W(s)\right)^{p}+C\left(\sup _{[0, t]} W(s)\right)^{(p+1) / 2} .
$$

Define

$$
M(t)=\sup _{[0, t]} W(s)
$$


Consequently, inequality (6.50) can be rewritten as

$$
M(t) \leq C\left(I_{0}+M(t)^{p}+M(t)^{p+1}\right)
$$

and we conclude by standard arguments (cf. [22]) that for sufficiently small $I_{0}$, we have

$$
M(t) \leq C I_{0}, \quad \forall t \geq 0
$$

This yields

$$
\left\|e^{\phi(t, x)} D u(t)\right\|_{2}+(1+t)^{N / 4+1 / 2}\|D u(t)\|_{2} \leq C I_{0} .
$$

This proves Theorem 6.1.

\subsection{The subcritical case $1<p<1+\frac{4}{N}$}

In this subsection, we consider problem (6.1) in the subcritical case $1<p<1+\frac{4}{N}$ and inspired by the method introduced in [20], we prove the global existence and the decay properties of the solutions. Unlike the supercritical case, here we prove our result without assuming any smallness assumption on the initial data. Let us assume first that

$$
I_{0}^{2}:=\int_{\mathbb{R}^{N}} e^{\beta|x|^{2}}\left(\left|u_{1}(x)\right|^{2}+\left|\nabla u_{0}(x)\right|^{2}+\left|u_{0}(x)\right|^{2}+\left|u_{0}(x)\right|^{p}\right) d x<+\infty
$$

for some $\beta>0$. Then, we have:

Theorem 6.12 Assume that $1<p \leq 1+4 / N$. Let $\left(u_{0}, u_{1}\right) \in H^{1}\left(\mathbb{R}^{N}\right) \cap L^{2}\left(\mathbb{R}^{N}\right)$ such that (6.54) holds. Then any solution of (6.1) satisfies for all $t \geq 0$

$$
\begin{aligned}
& \text { (i) }\|u(t, .)\|_{2} \leq C I_{0}(1+t)^{-\frac{1}{p-1}+\frac{N}{4}}, \\
& \text { (ii) }\|u(t, .)\|_{p+1} \leq C I_{0}^{2 /(p+1)}(1+t)^{-\frac{2}{(p-1)(p+1)}+\frac{N}{2(p+1)},} \\
& \text { (iii) }\|\nabla u(t, .)\|_{2}+\left\|u_{t}(t, .)\right\|_{2} \leq C I_{0}(1+t)^{-\frac{1}{p-1}+\frac{N}{4}}, \\
& \text { (iv) }\|u(t, .)\|_{1} \leq C I_{0}(1+t)^{-\frac{1}{p-1}+\frac{N}{2}},
\end{aligned}
$$

where $C$ is a positive constant.

Proof. In this case, the weight function $\phi$ can be taken similarly as before

$$
\phi(t, x)=\frac{a|x|^{2}}{4\left(t+t_{0}\right)}, \quad a>0, \text { and } t_{0} \geq 1 .
$$

Now, it is clear that from (6.28) we can choose suitable constants $a, \mu_{1}, \mu_{2}$ and $\varepsilon$ such that there exists $\lambda_{1}>0$ satisfying

$$
\frac{d}{d t} \int_{\mathbb{R}^{N}} \mathscr{L}(t) d x+\mathscr{F}(t) \leq 0, \quad \forall t>0
$$

where

$$
\mathscr{F}(t)=\lambda_{1} \int_{\mathbb{R}^{N}} e^{2 \phi}\left(\left|u_{t}\right|^{2}+|\nabla u|^{2}+|\nabla \phi|^{2} u^{2}+(g \diamond \nabla u)(t)+|u|^{p+1}\right) d x .
$$


Let us for example choose $a, \mu_{1}, \mu_{2}$ and $\varepsilon$ in (6.28) as follows:

As in the previous section, we take $\eta=l /(1-l), \mu_{2}=4 a$ and $\mu_{1}=a$ in (6.28) and after that we write $\phi_{t}=-\frac{1}{a}|\nabla \phi|^{2}$, and fix $\varepsilon$ small such that

$$
\varepsilon<\min \left(1 / 8, \frac{\zeta l}{1-l}\right)
$$

Then, we take $a$ small enough such that

$$
a<\min \left(l / 16, \frac{\varepsilon l}{2(1-l)}, \frac{\zeta l}{(1-l)(4 \varepsilon+1)}\right) .
$$

Now, define

$$
\overline{\mathscr{L}}(t)=\int_{\mathbb{R}^{N}} \mathscr{L}(t) d x
$$

and

$$
\overline{\mathscr{E}}(t)=\int_{\mathbb{R}^{N}} e^{2 \phi}\left(\left|u_{t}\right|^{2}+|\nabla u|^{2}+u^{2}+(g \diamond \nabla u)(t)+|u|^{p+1}\right) d x .
$$

Then from (6.31), we obtain

$$
\beta_{1} \overline{\mathscr{E}}(t) \leq \overline{\mathscr{L}}(t) \leq \beta_{2} \overline{\mathscr{E}}(t), \quad \forall t \geq 0
$$

For $\rho>0$, we multiply (6.56) by $\left(t_{0}+t\right)^{\rho}$ to obtain

$$
\frac{d}{d t}\left\{\left(t_{0}+t\right)^{\rho} \overline{\mathscr{L}}(t)\right\}+\left(t_{0}+t\right)^{\rho}\left(\mathscr{F}(t)-\frac{\rho}{t_{0}+t} \overline{\mathscr{L}}(t)\right) \leq 0 .
$$

By using (6.59), the last term in the left hand side of (6.60) can be estimated as follows:

$$
\begin{aligned}
\mathscr{F}(t)-\frac{\rho}{t_{0}+t} \overline{\mathscr{L}}(t) \geq & \mathscr{F}(t)-\frac{\rho \beta_{2}}{t_{0}+t} \overline{\mathscr{E}}(t) \\
\geq & \left(\frac{\lambda_{1}}{2}-\frac{\rho \beta_{2}}{t_{0}}\right) \int_{\mathbb{R}^{N}} e^{2 \phi}\left(\left|u_{t}\right|^{2}+|\nabla u|^{2}+(g \diamond \nabla u)(t)+|u|^{p+1}\right) d x \\
& -\frac{\rho \beta_{2}}{t_{0}+t} \int_{\mathbb{R}^{N}} e^{2 \phi} u^{2} d x \\
= & I_{1}+I_{2} .
\end{aligned}
$$

By choosing $t_{0}$ large enough such that $t_{0}>\frac{4 \rho \beta_{2}}{\lambda_{1}}$, then we obtain

$$
\begin{aligned}
I_{1} \geq & \frac{\lambda_{1}}{4} \int_{\mathbb{R}^{N}} e^{2 \phi}\left(\left|u_{t}\right|^{2}+|\nabla u|^{2}+(g \diamond \nabla u)(t)+|u|^{p+1}\right) d x \\
& :=\frac{\lambda_{1}}{4} \hat{\mathscr{E}}(t) .
\end{aligned}
$$

To estimate $I_{2}$, let us denote

$$
\Omega_{\kappa}:\left\{x \in \mathbb{R}^{N}: \kappa|x| \geq \sqrt{t+t_{0}}\right\}, \quad \text { and } \quad \Omega_{\kappa}^{c}:=\mathbb{R}^{N} \backslash \Omega_{\kappa},
$$

where $\kappa=a \sqrt{\frac{\lambda_{1}}{8 \rho \beta_{2}}}$. 
By denoting

$$
\frac{\rho \beta_{2}}{t_{0}+t} \int_{\mathbb{R}^{N}} e^{2 \phi} u^{2} d x=\int_{\Omega_{K}}+\int_{\Omega_{K}^{c}}=I_{21}+I_{22},
$$

then we have

$$
\begin{aligned}
I_{21} & \leq \frac{\rho \beta_{2}}{t_{0}+t} \int_{\Omega_{\kappa}} \frac{\kappa^{2}|x|^{2}}{t_{0}+t} e^{2 \phi} u^{2} d x \\
& \leq \frac{4 \rho \kappa^{2} \beta_{2}}{a^{2}} \int_{\mathbb{R}^{N}} \frac{a^{2}|x|^{2}}{4\left(t_{0}+t\right)^{2}} e^{2 \phi} u^{2} d x=\frac{\lambda_{1}}{2} \int_{\mathbb{R}^{N}} e^{2 \phi}|\nabla \phi|^{2} u^{2} d x .
\end{aligned}
$$

Concerning $I_{22}$, we have

$$
I_{22}=\frac{\rho \beta_{2}}{t_{0}+t} \int_{\Omega_{K}^{c}} e^{2 \phi \cdot \frac{p-1}{p+1}} \cdot e^{2 \phi \cdot \frac{2}{p+1}} u^{2} d x
$$

and using Young's inequality with $\frac{1}{\frac{p+1}{p-1}}+\frac{1}{\frac{p+1}{2}}=1$, we get

$$
\begin{aligned}
I_{22} & \leq \frac{\lambda_{1}}{2} \int_{\mathbb{R}^{N}} e^{2 \phi}|u|^{p+1} d x+C \int_{\Omega_{K}^{c}}\left(t_{0}+t\right)^{-\frac{p+1}{p-1}} e^{\frac{a|x|^{2}}{2\left(t_{0}+t\right)}} d x \\
& \leq \frac{\lambda_{1}}{2} \int_{\mathbb{R}^{N}} e^{2 \phi}|u|^{p+1} d x+C\left(t_{0}+t\right)^{-\frac{p+1}{p-1}} \int_{\Omega_{K}^{c}} d x \\
& \leq \frac{\lambda_{1}}{2} \int_{\mathbb{R}^{N}} e^{2 \phi}|u|^{p+1} d x+C\left(t_{0}+t\right)^{-\frac{p+1}{p-1}+\frac{N}{2}} .
\end{aligned}
$$

Consequently, combining (6.61) with (6.62)-(6.64), we find

$$
\mathscr{F}(t)-\frac{\rho}{t_{0}+t} \overline{\mathscr{L}}(t) \geq \frac{\lambda_{1}}{4} \hat{\mathscr{E}}(t)-C\left(t_{0}+t\right)^{-\frac{p+1}{p-1}+\frac{N}{2}} .
$$

Inequality (6.60) together with (6.65) yield

$$
\frac{d}{d t}\left\{\left(t_{0}+t\right)^{\rho} \overline{\mathscr{L}}(t)\right\}+\frac{\lambda_{1}}{4}\left(t_{0}+t\right)^{\rho} \hat{\mathscr{E}}(t) \leq C\left(t_{0}+t\right)^{\rho} \cdot\left(t_{0}+t\right)^{-\frac{p+1}{p-1}+\frac{N}{2}} .
$$

Next, for $0<\varepsilon<1$, we choose $\rho$ such that

$$
\rho-\frac{p+1}{p-1}+\frac{N}{2}=-1+\varepsilon
$$

that is for

$$
\rho=\left(\frac{p+1}{p-1}-\frac{N}{2}\right)-1+\varepsilon .
$$

In order to get $\rho>0$ in (6.67), for any $\varepsilon>0$, we have to assume that $p \leq 1+4 / N$.

Thus, integrating (6.66), over $[0, t]$ and using (6.59), we obtain

$$
\begin{aligned}
\beta_{1}\left(t_{0}+t\right)^{\rho} \overline{\mathscr{E}}(t)+\frac{\lambda_{1}}{4} \int_{0}^{t}\left(t_{0}+\tau\right)^{\rho} \hat{\mathscr{E}}(\tau) d \tau & \leq\left(t_{0}\right)^{\rho} \overline{\mathscr{L}}(0)+C \int_{0}^{t}\left(\tau+t_{0}\right)^{-1+\varepsilon} d \tau \\
& \leq \beta_{2}\left(t_{0}\right)^{\rho} \overline{\mathscr{E}}(0)+C_{\varepsilon}\left(t+t_{0}\right)^{\varepsilon}
\end{aligned}
$$


Dividing both sides in (6.68) by $\left(t+t_{0}\right)^{\varepsilon}$, we obtain

$$
\overline{\mathscr{E}}(t) \leq C(t+1)^{-\frac{2}{p-1}+\frac{N}{2}},
$$

which implies the first three estimates in (6.55). To prove the last last estimate in (6.55), we have from (6.69) and Lemma 6.6

$$
\begin{aligned}
\|u(t)\|_{1} & \leq\left(\int_{\mathbb{R}^{N}} e^{-2 \phi(t, x)} d x\right)^{1 / 2}\left(\int_{\mathbb{R}^{N}} e^{2 \phi(t, x)} u^{2}(t, x) d x\right)^{1 / 2} \\
& \leq C(1+t)^{-\frac{1}{p+1}+\frac{N}{2}} .
\end{aligned}
$$

This completes the proof of Theorem 6.12.

\section{Concluding remarks}

In this section we conclude with few remarks, pointing out some open problems and future directions worth pursuing.

Remark 7.1 It is well known that for the linear version of problem (1.3) in bounded domains $\Omega \subset \mathbb{R}^{N}$, the exponential (resp. polynomial) decay of $g$ is a sufficient condition for the exponential (resp. polynomial) decay of the solution $u$. It was shown in [5] that the exponential decay of $g$ is also a necessary condition for the exponential decay of $u$. This is somehow surprising, since the memory introduces dissipation and for $g=0$, the solution $u$ decays exponentially. It seems that the presence of the dissipation term $u_{t}$ is hidden by the presence of the memory term. The same situation seems to appear in $\mathbb{R}^{N}$. In fact the decay rate given in [4] for (1.3) is of order $(1+t)^{-N / 4}$ which is the same decay obtained in [1] for (1.3) without $u_{t}$.

Remark 7.2 The decay rate of the Cauchy problem

$$
u_{t t}-\Delta u+u_{t}=0
$$

with initial data

$$
\left(u_{0}, u_{1}\right) \in\left(H^{1}\left(\mathbb{R}^{N}\right) \cap L^{1}\left(\mathbb{R}^{N}\right)\right) \times\left(L^{2}\left(\mathbb{R}^{N}\right) \cap L^{1}\left(\mathbb{R}^{N}\right)\right)
$$

is

$$
\left\|u_{t}\right\|_{2}+\|\nabla u\|_{2} \leq C(1+t)^{-N / 4-1 / 2} .
$$

See [18]. According to Remark 7.1 and since the dissipative term induced by the memory term is weaker than the frictional damping term $u_{t}$, it is natural to see that the solution of the linear problem of (1.3) with the same initial data (7.2) decays as

$$
\left\|u_{t}\right\|_{2}+\|\nabla u\|_{2} \leq C(1+t)^{-N / 4} .
$$

Remark 7.3 As, we have said before, the exponential (resp. polynomial) decay of the kernel is necessary condition to obtain an exponential (resp. polynomial) decay of the solution of (1.3). recently Pata [21] has obtained some decay results by allowing the kernel $g$ to be flat provided that the set

$$
\mathscr{F}_{g}=\left\{s \in \mathbb{R}^{+}: g(s)>0 \text { and } g^{\prime}(s)=0\right\}
$$

where the kernel $g$ is flat is sufficiently small.

\section{Acknowledgments}

The second author was supported by the DFG project RA 504/3-3. This author wishes to thank the Department of Mathematics and Statistics, University of Konstanz for its financial support and its kind hospitality. 


\section{References}

[1] M. Conti, S. Gatti, and V. Pata. Decay rates of Volterra equations on $\mathbb{R}^{n}$. Cent. Eur. J. Math., 5(4):720$732,2007$.

[2] C. M. Dafermos. Asymptotic stability in viscoelasticity. Arch. Rational Mech. Anal., 37:297-308, 1970.

[3] G. Dassios and F. Zafiropoulos. Equipartition of energy in linearized 3-d viscoelasticity. Quart. Appl. Math., 48(4):715-730, 1990.

[4] P. M. N. Dharmawardane, J. E. Muñoz Rivera, and S. Kawashima. Decay property for second order hyperbolic systems of viscoelastic materials. J. Math. Anal. Appl., 366:621-635, 2010.

[5] M. Fabrizio and S. Polidoro. Asymptotic decay for some differential systems with fading memory. Appl. Anal., 81(6):1245-1264, 2002.

[6] A. Haraux and F. B. Weissler. Nonuniqueness for a semilinear initial value problem. Indiana Univ. Math. J., 31:167-189, 1982.

[7] N. Hayashi, E. I. Kaikina, and P. I. Naumkin. Damped wave equation in the subcritical case. J. Differential Equations, 207:161-194, 2004.

[8] N. Hayashi, E. I. Kaikina, and P. I. Naumkin. Damped wave equation with supercritical nonlinearities. Differential and Integral Equations, 17:637-652, 2004.

[9] T. Hosono and S. Kawashima. Decay property of regularity-loss type and application to some nonlinear hyperbolic-elliptic system. Math. Mod. Meth. Appl. Sci., 16:1839-1859, 2006.

[10] W. J. Hrusa and J. A. Nohel. The Cauchy problem in one-dimensional nonlinear viscoelasticity. $J$. Differential Equations, 59:388-412, 1985.

[11] K. Ide and S. Kawashima. Decay property of regularity-loss type and nonlinear effects for dissipative Timoshenko system. Math. Mod. Meth. Appl. Sci., 18(7):1001-1025, 2008.

[12] R. Ikehata. New decay estimates for linear damped wave equations and its application to nonlinear problem. Math. Meth. Appl. Sci., 27(8):865-889, 2004.

[13] R. Ikehata, K. Nishihara, and H. Zhao. Global asymptotics of solutions to the Cauchy problem for the damped wave equation with absorption. J. Differential Equations, 226(1):1-29, 2006.

[14] R. Ikehata and K. Tanizawa. Global existence of solutions for semilinear damped wave equations in $\mathbb{R}^{N}$ with noncompactly supported initial data. Nonlinear Anal, 61:1189-1208, 2005.

[15] M. Kafini and S. A. Messoudi. On the uniform decay in viscoelastic problems in $\mathbb{R}^{n}$. Appl. Math. Comput., 215(3):1161-1169, 2009.

[16] G. Karch. Selfsimilar profiles in large time asymptotics of solutions to damped wave equations. Studia Math., 143(2):175-197, 2000.

[17] S. Kawashima, M. Nakao, and K. Ono. On the decay property of solutions to the Cauchy problem of the semilinear wave equation with a dissipative term. J. Math. Soc. Japan., 47(4):617-653, 1995. 
[18] A. Matsumura. On the asymptotic behavior of solutions of semi-linear wave equations. Publ. Res. Inst. Math. Sci., 12(1):169-189, 1976.

[19] T. Narazaki and K. Nishihara. Asymptotic behavior of solutions for the damped wave equation with slowly decaying data. J. Math. Anal. Appl., 338(2):803-819, 2008.

[20] K. Nishihara and H. Zhao. Decay properties of solutions to the Cauchy problem for the damped wave equation with absorption. J. Math. Anal. Appl., 313(2):598-610, 2006.

[21] V. Pata. Exponential stability in linear viscoelasticity. Quart. Appl. Math., 64(3):499-513, 2006.

[22] R. Racke. Lectures on nonlinear evolution equations. Initial value problems. Aspects of mathematics, E19. Friedrich Vieweg and Sohn: Braunschweig, Wiesbaden, 1992.

[23] J. E. Muñoz Rivera. Asymptotic behaviour in linear viscoelasticity. Quart. Appl. Math., 52(4):628648, 1994.

[24] J. E. Muñoz Rivera, M. Naso, and E. Vuk. Asymptotic behavior of the energy for electromagnetic system with memory. Math. Meth. Appl. Sci., 25(7):819-841, 2004.

[25] J.E. Muñoz Rivera and E. C. Lapa. Decay rates of solutions of an anisotropic inhomogeneous $n$ dimensional viscoelastic equation with polynomially decaying kernels. Comm. Math. Phys., 177:583$602,1996$.

[26] B. Said-Houari. Convergence to strong nonlinear diffusion waves for solutions to $p$-system with damping. J. Differential Equations, 247:917-930, 2009.

[27] G. Todorova and B. Yordanov. Critical exponent for a nonlinear wave equation with damping. $J$. Differential Equations, 174:464-489, 2001. 